\title{
On the automatic and $a$ priori design of unstructured mesh resolution for coastal ocean circulation models
}

\author{
Keith J. Roberts ${ }^{1}$, William J. Pringle ${ }^{1 *}$, Joannes J. Westerink ${ }^{1}$, Marite Teresa Contreras ${ }^{1}$, \\ Damrongsak Wirasaet ${ }^{1}$ \\ ${ }^{1}$ Department of Civil and Environmental Engineering and Earth Sciences, University of Notre
} Dame, Notre Dame, IN

\section{Key Points:}

- The U.S. East Coast and Gulf Coast domain is automatically discretized with a 50-m minimum mesh size.

- A priori mesh size functions based on shoreline geometry and seabed topography are used.

- A sequence of mesh designs systemically analyze the response of surface tides to mesh size distribution.

- Recommendations of mesh size function combinations and parameters to efficiently and accurately discretize the domain are presented. 


\section{Abstract}

This study investigates the design of unstructured mesh resolution and its impact on the modeling of barotropic tides along the United States East Coast and Gulf Coast (ECGC). A discrete representation of a computational ocean domain (mesh design) is necessary due to finite computational resources and an incomplete knowledge of the physical system (e.g., shoreline and seabed topography). The selection of mesh resolution impacts both the numerical truncation error and the approximation of the system's physical domain. To increase confidence in the design of high-resolution coastal ocean meshes and to quantify the efficacy of current mesh design practices, an automated mesh generation approach is applied to objectively control resolution placement based on a priori information such as shoreline geometry and seabed topographic features. The simulated harmonic tidal elevations for each mesh design are compared to that of a reference solution, computed on a 10.8 million vertex mesh of the ECGC region with a minimum shoreline resolution of 50-m. Our key findings indicate that existing mesh designs that use uniform resolution along the shoreline and slowly varying resolution sizes on the continental shelf inefficiently discretize the computational domain. Instead, a targeted approach that places fine resolution in narrow geometric features, along steep topographic gradients, and along pronounced submerged estuarine channels, while aggressively relaxing resolution elsewhere, leads to a mesh with an order of magnitude fewer vertices than the reference solution with comparable accuracy (within 3\% harmonic elevation amplitudes in $99 \%$ of the domain).

\section{Introduction and background}

Two-dimensional (2D) unstructured triangular meshes are widely used to represent the horizontal domain in the simulation of hydrodynamic processes of ocean, shelf and inland coastal water systems. In general, these variable resolution meshes are used to study a broad spectrum of processes in the coastal ocean from wind waves with periods on the order of seconds to large scale shelf and oceanic circulation with timescales on the order of days to months. Most commonly, barotropically-driven long wave processes (tides, surge, and tsunami) with periods on the order of minutes to hours are simulated with these meshes. This includes the modeling of tidal dynamics [Blanton et al., 2004; Chen et al., 2011; Pringle et al., 2018a] and the prediction of extreme water levels during high energy events such as tropical and extratropical storms [Westerink et al., 2008; Dietrich et al., 2010, 2011; Beardsley et al., 2013; Chen et al., 2013; Hope et al., 2013; Xu et al., 2013; Zheng et al., 2013; Xie et al., 2016; Cyriac et al., 2018]. Critically, unstructured triangular meshes facilitate seamless cross-scale modeling of the complete long wave spectrum [Zhang and Baptista, 2008; Zhang et al., 2016; Pringle et al., 2019].

Unstructured meshes are used to capture the detailed hydrodynamic response driven by the governing physical processes and their interactions with the physical system. Historically in fluid mechanics, approaches to mesh design and adaption have often been based on a posteriori techniques based on the residual of the flow solution on a per element basis [e.g. Oden et al., 1990; Behrens, 1998]. In coastal modeling, an a posteriori analysis has been performed using a formal local truncation error analysis [LTEA; Hagen et al., 2000, 2002; Parrish and Hagen, 2009] with the objective to equalize the truncation error throughout the computational domain. However, as finer mesh sizes are used to reduce the truncation error, new narrower shoreline details emerge that can alter the system's response and these aspects are difficult to incorporate into the error indicator. Thus, while the estimate of the numerical truncation error for a given initial mesh description can be minimized, the system domain error may persist because critical features still do not exist in the boundary description and these features may not be detectable by the error indicator.

The aforementioned considerations motivates us to use a feature-driven a priori approach. In fact, for the most part meshes for coastal modeling have been developed using an a priori approach adjusting resolution to match both the physical system's length scale and estimated length scales of the dominant physics [e.g., Lyard et al., 2006; Bunya et al., 2010; Chen et al., 2011; Luettich and Westerink, 2013; Kerr et al., 2013; Chen et al., 2016]. Feature-driven a priori approaches have been proposed to automatically design meshes in this manner [Bilgili et al., 2006; Conroy et al., 
2012; Roberts et al., 2018]. Nevertheless, until now it has been difficult to build a sufficient number of meshes to enable a controlled comparison of the simulated results for realistic coastal ocean hydrodynamic models through the traditional ad hoc and tedious [Hagen et al., 2001] development process. However, recent advances in automated unstructured mesh generation technology for the ocean [Remacle and Lambrechts, 2016; Engwirda, 2017; Candy and Pietrzak, 2018; Avdis et al., 2018; Roberts et al., 2018] now enable well-defined repeatable workflows for generating detailed multiscale coastal ocean meshes. These approaches alleviate the burden previously associated with the model development steps and ensure that the development process is sufficiently controlled to facilitate inter-comparisons between simulation results from a variety of mesh designs with logical perturbations.

A ubiquitous feature-driven a priori meshing criteria for coastal modeling is the wavelengthto-gridscale heuristic that sizes resolution according to an estimate of depth-dependent shallow water wave celerity to maintain constant discretization of the wavelength of the dominant mode [Westerink et al., 1994; Lyard et al., 2006; Greenberg et al., 2007; Westerink et al., 2008]. This heuristic produces meshes that contain the finest resolution nearshore, element size transitions that vary smoothly, and nearly constant resolution across the continental shelf. However, the wavelengthto-gridscale heuristic is based on a one-dimensional analysis that assumes no bathymetric gradients and thus cannot capture complexity of seabed features like shelf breaks and isolated banks [Greenberg et al., 2007] nor the intricacies of the 2D shoreline. Further, submarine channels that are important to convey flow into the estuarine system can become coarsely discretized with its application. While a long legacy of meshes have been built with this heuristic, the application of resolution using this approach leads to models with many degrees-of-freedom if the parameter dictating the number of nodes per wavelength is set to a large value to compensate for inadequately targeting resolution at the aforementioned features.

Consideration of the topographic-length scale, i.e., applying finer resolution directly proportional to the seabed depth and inversely proportional to seabed topographic gradient has also been widely conducted [Lyard et al., 2006; Chen et al., 2016; Engwirda, 2017]. This approach refines the resolution in proximity to the shelf break and submarine ridges and banks, which often tend to be co-located with large gradients in the solution [Hannah and Wright, 1995]. In fact, the LTEA analysis method proposed by Hagen et al. [2000, 2002] demonstrated that the minimization of truncation error tended to produce a distribution of vertices that resembled the application of the topographic-length scale. Representing steep gradients is also useful to capture submarine ridges and rough topography over which internal tides are generated [Garrett and Kunze, 2007]. This process is often included as a parameterized dissipation process in barotropic tidal models [Green and Nycander, 2013; Pringle et al., 2018a,b]. However, a drawback of the topographic-length scale is that on the inner shelf the topographic gradient to depth ratio can become large due to topographic irregularities which leads to excessively fine resolution as compared to the length scales of the dominant physics.

Unstructured meshes have a powerful capability to efficiently capture the geometrically complex form of the shoreline and of the complex esutaries and the connected dendritic inland channels, but most prior works have not taken full advantage of this capability by applying uniformly fine resolution along shorelines and within inland waterways in regions of interest. For instance, NOMAD (NOAA Operational Model with ADCIRC), a mesh used for real-time predictions of storm surge and tides (e.g., ASGS [Fleming et al., 2008]), uses uniform coastal resolution of approximately $250 \mathrm{~m}$ along all the United States East Coast and Gulf Coasts (ECGC) [Technology Riverside Inc. and AECOM, 2015]. Other examples of meshes that resolve the shoreline uniformly includes those used in recent long-term regional analyses of storm surge and tides in ECGC [ 1-5 km; Muis et al., 2019; Marsooli and Lin, 2018], and those used for hurricane-induced coastal flooding in the northern Gulf of Mexico [ 100 m; Kerr et al., 2013]. On one hand, uniform shoreline resolution ensures that the representation of the inlet/backbay system that control coastal inshore hydrodynamics is best represented in the mesh of the specified resolution. On the other hand, the application of nearly uniform resolution nearshore over-resolves many sections of the coastline and inland waters that are straight and geometrically simple leading to a situation where cost con- 
straints then necessitate under-resolving narrow and constricted waterways. Studies in the South Atlantic Bight have demonstrated that the representation of the estuary system as a whole can alter the morphodynamic feedback between the tides and the shoreline form [Blanton et al., 2004; Bacopoulos and Hagen, 2017]. Thus, beyond applying fine resolution zones nearshore, it is often critical to resolve the intricate dendritic inland waters and to quantify the feedback effects from the integrated system. These irregular shoreline and inland systems can be efficiently captured using highly variable mesh resolution.

Another consideration for developing unstructured meshes is the rate of element size transitions between zones of variable resolution otherwise referred to as the gradation [Persson, 2006]. It is known that element size transitions must be smooth and bounded above by a constant to avoid numerical errors and inaccuracies [Shewchuk, 2002; Bilgili et al., 2006]. In fact, the error analysis undertaken by Hagen et al. [2000] clearly demonstrates that a gradation above 50\% will cause odd order error terms to dominate and subsequently degrade a formally second order numerical method to first order. While a theoretical upper bound value for the gradation is known, the total number of vertices in a coastal ocean discretization can wildly vary depending on the choice of gradation below 50\% (a large gradation will lead to fewer vertices). Thus, the gradation rate needs to be explored to identify a suggested tighter range of values that efficiently discretizes the physical domain while maintaining accuracy in the simulation of the coastal ocean.

A common first step in the production of a coastal hydrodynamic model is to assess the simulated accuracy of astronomical tides [e.g., Pringle et al., 2018a] prior to the simulation of extreme sea levels. At this initial stage of the model development process, the model is calibrated through adjustments to frictional and dissipative parameterizations in order to agree with measured data. However, when the mesh underresolves shoreline and seabed features, the system's response may become distorted leading to an inability to correctly produce solutions across the entire domain and energy spectrum. An example of this would be tuning the model to agree with observations of dominant semi-diurnal elevation tidal constituent regionally but this may not lead to a good agreement globally nor for the other tidal constituents. Instead, by gathering knowledge on how tidal solution depends on mesh resolution in realistic coastal modeling problems, we can enable efficient and uniformly more accurate mesh designs that can then facilitate more dynamically correct calibrations of friction parameterizations.

Our premise is that the numerical modeling of the circulation and flow of water is largely driven and controlled by the representation of the physical system and the representation of the physical system is integrally related to the mesh sizing functions. Thus, the sizing functions need to be carefully considered for ensuring high fidelity coastal ocean hydrodynamic simulations that have a relatively low associated computational cost. This is particularly relevant for operational/realtime forecast systems in order to be practically computationally feasible. Many of the previously used a priori mesh size heuristics (e.g., topographic-length scale, and distance-to-shoreline) have proven useful in practice for producing accurate solutions for tides and storm surges. Thus, we have devised an approach that combines and builds on such mesh size heuristics to variably resolve shoreline geometry, seabed topography, controlling the geometric expansion of element sizes, and capturing submarine channels that convey flow into and out of the estuaries. Our ultimate goal is to capture the physical system and response with the fewest number of degrees of freedom while preserving the performance of the solution compared to measured data. Here, we apply our approach to the widely studied ECGC region and conduct an in-depth analysis of the sensitivity of the barotropic tides to the domain discretization.

This paper addresses the following two questions:

a) How does the simulation of barotropic tides respond to the representation of shoreline geometry and seabed topography in the ECGC region? What are the sources of error and how do these contribute to the measured differences?

b) Can we incorporate our results from a) to make recommendations for a set of mesh size functions that place resolution according to shoreline geometry and seabed topography to 


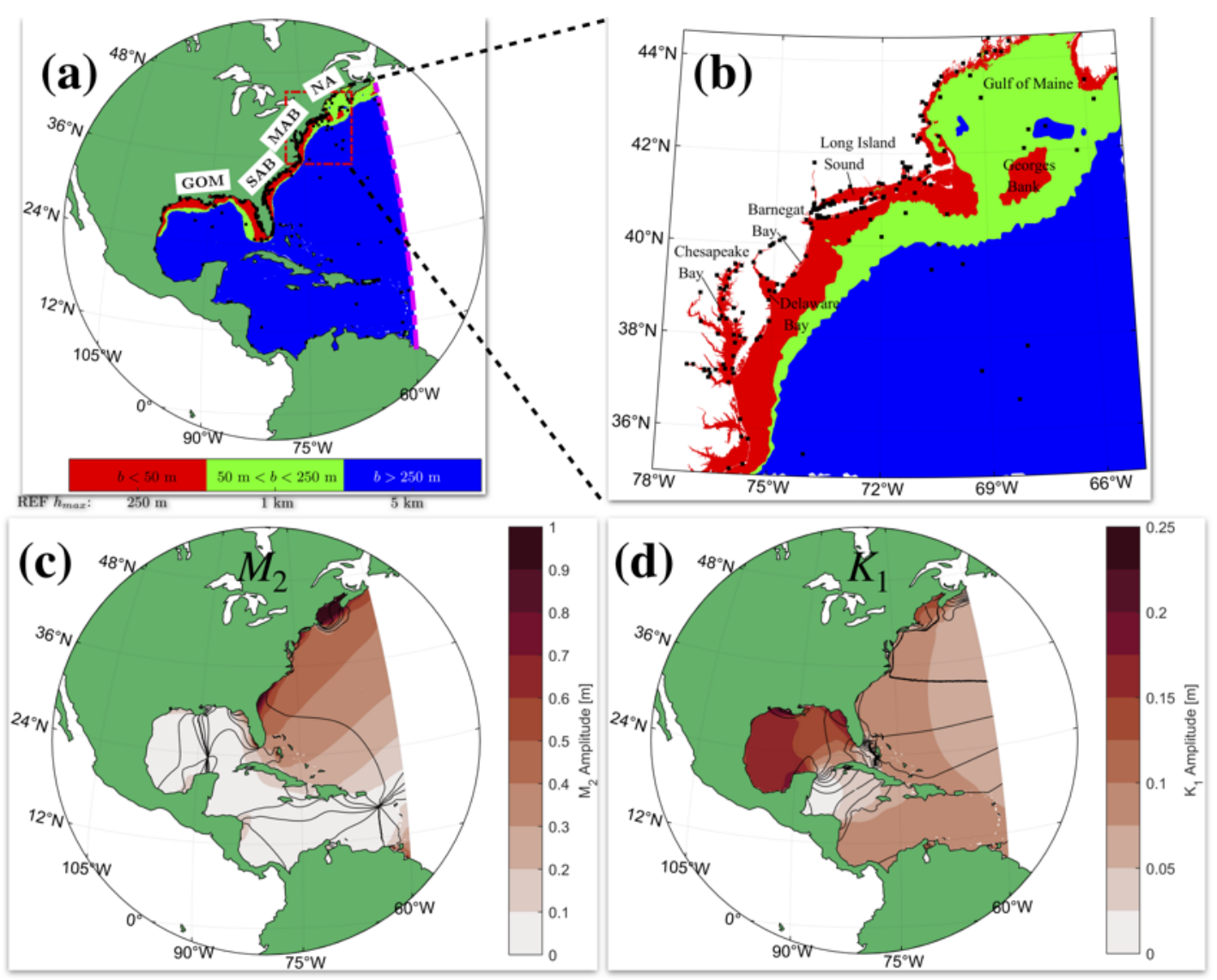

Figure 1. The study area in which colored zones in the top panels indicate the mesh size upper bounds $\left(h_{\max }\right)$ in the reference $(\mathrm{REF})$ mesh (minimum mesh size is $L_{\min }=50 \mathrm{~m}$ ). The red and green colored zones together indicate the comparison zones for all the cumulative area fraction error curve calculations. The dashed magenta line indicates the open ocean boundary on which tidal elevations are specified. The bottom left and right panels indicate TPXO9.1 solutions of the $\mathrm{M}_{2}$ and $\mathrm{K}_{1}$ tidal constituent elevation amplitudes (colors) and phase contours in intervals of $30^{\circ}\left(\mathrm{M}_{2}\right)$ and $15^{\circ}\left(\mathrm{K}_{1}\right)$.

efficiently discretize coastal ocean domains that approximately reproduce simulation results from an extremely well-resolved mesh?

\section{Methods, Data and Tools}

\subsection{ECGC Study Domain and Data}

The ECGC study domain for this work (Figure 1) contains a single open ocean boundary along the $60^{\circ} \mathrm{W}$ meridian which is placed here for geometric simplicity and because it lies in the deep ocean where the tides vary gradually and hence suitable for coupling to global tidal model solutions that are highly accurate in the deep ocean [Stammer et al., 2014]. The placement of the open boundary in this way is sufficiently far from the coastal zones to represent tide responses throughout the ECGC domain [Westerink et al., 1994].

The domain is classified into four distinct regions as shown in Figure 1 along with co-tidal and co-amplitude lines of the dominant constituents. The tides are predominately semi-diurnal dominated by the $\mathrm{M}_{2}$ along the Eastern Coast of the United States - North Atlantic (NA), MidAtlantic Bight (MAB), and South Atlantic Bight (SAB). In the western half of the Gulf of Mexico 
(GOM) the $\mathrm{K}_{1}$ and $\mathrm{O}_{1}$ dominate water level variations, while the eastern side is mixed-diurnal with the $\mathrm{M}_{2}, \mathrm{~K}_{1}, \mathrm{O}_{1}$, and $\mathrm{S}_{2}$ contributing roughly in equal parts.

\subsubsection{Bathymetric and Shoreline Datasets}

The bathymetric data used for this study are primarily based on SRTM15+ [Sandwell et al., 2014] and supplemented in areas of overlap with the Coastal Relief Model [CRM; Amante and Eakins, 2009] in addition to local $1 / 3$ and 1/9 arc-sec NCEI topo-bathymetric coastal elevation model datasets where available (https://www.ngdc.noaa.gov/mgg/coastal/coastal.html). The entire bathymetric dataset was integrated into a final digital elevation model (DEM) that was re-sampled on a uniform grid spacing of 3 arc-sec $(\sim 90 \mathrm{~m})$, which is equal to the resolution of the CRM. For SRTM15+ and the CRM, the vertical uncertainty in the data is generally larger than the discrepancy between local mean sea level and the NAVD88 vertical reference datums, so no effort was made to rectify the vertical datum for these data. However, all NCEI local and regional datasets were adjusted to local mean sea level using VDatum [White and Hess, 2016] where the transformation was available. The horizontal datum of the re-sampled DEM is in geographic coordinates or WGS84.

Since the shoreline (where land meets the ocean in the temporal mean sense) as it exists in nature has a fractal geometry and is constantly evolving due to sedimentation and erosional processes, variations in discharge, sea level rise, and anthropomorphic effects, its exact representation may be intractable. For the purposes of this work, we consider a static version of the shoreline as depicted from the relatively recent (5-10 years old) topo-bathymetric data used in this study. A polyline that approximates the local mean sea level shoreline was extracted using the GRASS Geographical Information Systems r.contour module with a cut parameter of 150 [GRASS Development Team, 2017]. While higher quality shoreline vector datasets exist, a preference was given to the shoreline extracted from the re-sampled DEM that was created for this work given that it would produce mesh boundaries that are aligned with the $0-\mathrm{m}$ contour from the data sources. In other words, this helps to improve the agreement with the location of where the shoreline is when topobathymetric data is interpolated onto the mesh vertices. The discrete shoreline extracted from the DEM model can only resolve shoreline length-scales down to its horizontal resolution of 3 arc-sec (approximately $90 \mathrm{~m}$ ).

\subsubsection{Tide Gauge Data}

Harmonic tidal constituent observations at tide gauges in ECGC (Figure 1) are used in this study to the validate the model simulations on selected meshes. The observations are predominantly made up of posted harmonic constituents at 636 National Oceanic and Atmospheric Administration (NOAA) coastal tide gauges (https://tidesandcurrents.noaa.gov/stations.html?type= Harmonic+Constituents). An additional 31 observations located on the continental shelf and in deep water [Stammer et al., 2014] are also included (available from ftp://ftp.legos.obs-mip.fr/pub/ FES2012-project/data/gauges/2013-12-16/).

\subsection{Hydrodynamic Model Configuration}

This study uses the ADvanced CIRCulation model (ADCIRC) [Luettich and Westerink, 2004; Westerink et al., 2008] to perform the hydrodynamic simulations of two-dimensional (2D) barotropic tides. ADCIRC is a continuous-Galerkin finite element model that solves the primitive continuity equation using the so-called Generalized Wave Continuity Equation [GWCE; Lynch and Gray, 1979; Kinnmark, 1988] and a depth-averaged momentum equation on an unstructured triangular mesh [Westerink et al., 1992]. It is formally a second-order solver that discretizes the domain with linear elements.

We perform all simulations with the following setup: the model is forced by astronomical tidal elevation open ocean boundary conditions, astronomical tidal equilibrium potential terms, and astronomical tidal self-attraction and loading (SAL) terms [Hendershott, 1972]. 
In the ADCIRC solver, the time and space advective components of the equations can be excluded from calculations for numerical stability purposes; however, all terms were included in the calculations. Further wetting/drying was enabled although a minimum depth is enforced on the shoreline of $1 \mathrm{~m}$ below sea level to ensure flow through narrow channels on the scale of the minimum resolution. A constant quadratic bottom friction was used with the standard coefficient of 0.0025 . Horizontal dissipation was parameterized through a constant lateral eddy viscosity term of $50 \mathrm{~m}^{2} \mathrm{~s}^{-1}$. The GWCE mass matrix is solved using an explicit time discretization with mass lumping instead of the consistent implicit method. This choice was not found to affect the simulation results at the 2 second simulation timesteps we are using here with the Courant-limited explicit timestepping scheme. Therefore, the explicit method was preferred due to improved computationally efficiency (approximately twice as fast) [Tanaka et al., 2011].

\subsection{Mesh Generation}

The construction of regional coastal ocean meshes for hydrodynamic simulations in models such as ADCIRC is an involved process with many degrees of variation. In order to analyze how mesh resolution may affect numerical simulations, it is vital to have an automated and reproducible workflow to systematically control aspects of the mesh design. By reproducible we mean that given the exact same inputs and options, the vertex locations of a new instance of the mesh will be approximately the same having vertex/elemental densities within a fraction of the target density function and leading to negligible differences between simulation results repeated on various instances of the mesh. The approximate similarity of meshes is evidenced in results throughout the manuscript: nearly similar mesh designs exhibit the smallest relative differences between their solutions.

Some approaches and tools have been developed recently to make these workflows feasible [Engwirda, 2017; Gorman et al., 2008; Candy and Pietrzak, 2018; Roberts et al., 2018]. For this work, all unstructured meshes were developed with the OceanMesh $2 D$ software [Roberts et al., 2018; Roberts and Pringle, 2018]. OceanMesh2D is a self-contained MATLAB mesh generation toolkit for the development of 2D unstructured triangular meshes. Specifically, we use Version 2.0 of the software which is an extension of V1.0 [Roberts et al., 2018] with support for mesh generation using map projections to ensure that meshes on the sphere conform to Earth's curvature and obey user-defined resolution requests which are specified in meters. Any map projection that is featured in the $\mathrm{m} \_$map mapping package [Pawlowicz, 2018] can be selected.

A number of meshes are automatically generated in Lambert conformal conic projection space using the multiscale meshing approach [Roberts et al., 2018], whereby multiple boxes are used to cover the region roughly indicated by the green and red colored zones in Figure 1(a)-(b). Inside these boxes, the minimum resolution $L_{\min }$ is specified to between $50 \mathrm{~m}$ and $250 \mathrm{~m}$, depending on the experiment (see Section 2.4). A larger box covering the whole study region is used to mesh the rest of the domain with a minimum resolution of $1 \mathrm{~km}$ that is placed uniformly along the shoreline. The result is one seamless unstructured mesh, in which the software automatically smooths mesh resolution sizes between regions.

Topo-bathymetric data, available on a structured grid (DEM), is interpolated onto the mesh vertices using the grid-scale averaging approach that is built into the mesh generation software [Roberts et al., 2018]. Grid-scale averaging is used to minimize aliasing of the seabed topography on the mesh vertices that would other arise from curve-fitting interpolation schemes (e.g., linear interpolation). The minimization of sub-grid scale topo-bathymetric features in the interpolated seabed topography is important in order to study the effect of mesh resolution on the solution.

\subsection{Experimental Design}

In Sections 3.1 to 3.4 five experiments are explored to examine the effects of targeted placement of mesh resolution at various seabed and shoreline features according to a mesh size function or constraint (Table 1). Within each experiment three meshes (categorized as 'fine', 'medium', and 
'coarse' resolution) are generated by varying a single mesh size function parameter while holding all other parameters constant. Note that the variation of mesh sizing parameters is a multi-faceted problem and all the parameters interact (e.g., one parameter's value can mask effects of another). For example, a relatively higher feature size may cause finer resolution in deep offshore features that can be largely influential on the simulation of tides, as later shown. All meshes require a minimum mesh size and an element-to-element mesh size gradation rate (henceforth referred to as gradation), which are set to 50-m uniformly along the shoreline and $15 \%$, respectively, unless otherwise stated. The maximum mesh size is set to $10 \mathrm{~km}$ for all meshes.

The effect of the mesh size functions on the resulting triangulation's that are used in the various experiments (Table 1) are graphically illustrated in Figure 2, and described below:

- In the distance function (Figure 2(a)), mesh resolution is dictated by the minimum mesh size at the shoreline $\left(L_{\min }\right)$ and the maximum allowable expansion rate $(g)$. The variation of $L_{\text {min }}$ forms Experiment 1.

- The feature size function (Figure 2(b)) places mesh resolution according to the width of the geometric feature. The width is estimated as two times the sum of the distance from a point in the computational domain to the nearest shoreline point plus the distance from the same point to the nearest medial axis (Figure 2(c)). Varying the number of elements per geometric feature width forms Experiment 2.

- The gradation function bounds the mesh size transitions on the structured grid that the mesh size function is calculated on, which will determine the gradation $(g)$ on the mesh's triangulation. The variation of this parameter only forms Experiment 3.

- The slope function (Figure 2(e)) places mesh resolution according to the length of a topographic feature, targeting regions of high topographic gradients such as the continental shelf break and slope. Experiment 4 varies the number of elements per topographic length-scale.

- The submarine channel function (Figure 2(d)) targets mesh resolution along and near welldefined submarine channels such as dredged shipping channels or morphodynamic conveyances within estuaries that are identified through an upslope area calculation using a 1,000 DEM cell minimum threshold in Geographical Information Systems software. Experiment 5 varies the number of elements per channel width. The channel width is estimated according to the seabed depth near the channel and an assumed slope angle of $30^{\circ}$ with the seabed floor [see Roberts and Pringle, 2018].

A highly-refined reference (REF) mesh (Table 1) was generated to act as a proxy for the 'true' solution against which our meshes in the experiments are compared. In this mesh, a set of depth-based maximum element size constraints were used and a mesh size gradation of $15 \%$. Specifically, the minimum mesh resolution is $50-\mathrm{m}$ and the maximum resolution was bounded above by $250 \mathrm{~m}$ nearshore (depth, $b<50 \mathrm{~m}), 1 \mathrm{~km}$ on the continental shelf $(50 \mathrm{~m}<b<250 \mathrm{~m}$ ), and $5 \mathrm{~km}$ in the deep ocean $(b>250 \mathrm{~m})$. These mesh size constraints are conservative and they represent values that could be accommodated in terms of the total computational cost, Courantbased stability constraint, and the resolution of the geospatial data used $(\sim 90 \mathrm{~m})$. The REF mesh contains $N=10,746,955$ vertices and represents a mesh design that we classify as 'overly-discretized' in the sense that as this study will later demonstrate, it is possible to substantially reduce the vertex count while maintaining solution accuracy. It is important to note that for all mesh configurations the REF mesh is indeed finer than the other mesh designs except for the S20 mesh design in a local region on the Western side of the GOM.

Each mesh was used to perform a 122-day tidal simulation to assess the effects on the astronomical tides due to variations in mesh design. In these simulations, ADCIRC is forced through the tidal equilibrium potential and SAL terms throughout the domain and at the open ocean boundaries with four major semi-diurnal $\left(\mathrm{M}_{2}, \mathrm{~N}_{2}, \mathrm{~S}_{2}, \mathrm{~K}_{2}\right)$ and four major diurnal tidal constituents $\left(\mathrm{K}_{1}\right.$, $\mathrm{O}_{1}, \mathrm{P}_{1}, \mathrm{Q}_{1}$ ). Open boundary elevations are obtained from TPXO9.1 (http://volkov.oce.orst.edu/ tides/global.html) tidal solutions; SAL terms are obtained from FES2014 tidal loading solutions (ftp://ftp.legos.obs-mip.fr/pub/FES2012-project/data/LSA/FES2014/). In the assessment of the results of these simulations, a focus is placed primarily on the variation in the major semi-diurnal 


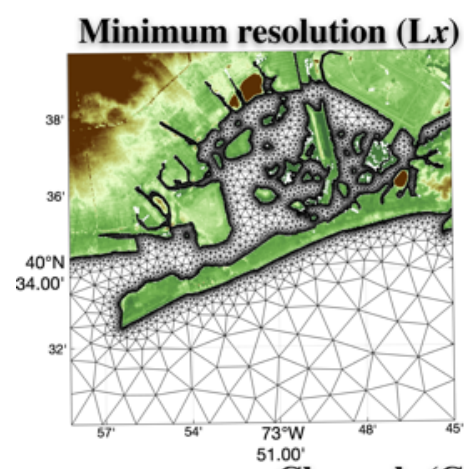

Channels (Cx)

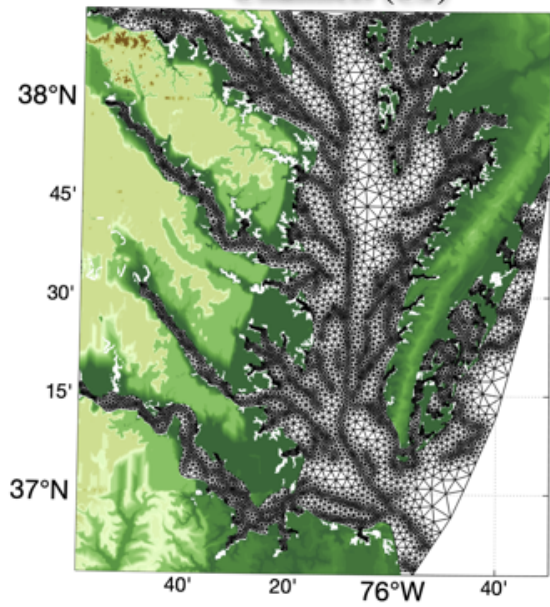

Feature size (FSx)
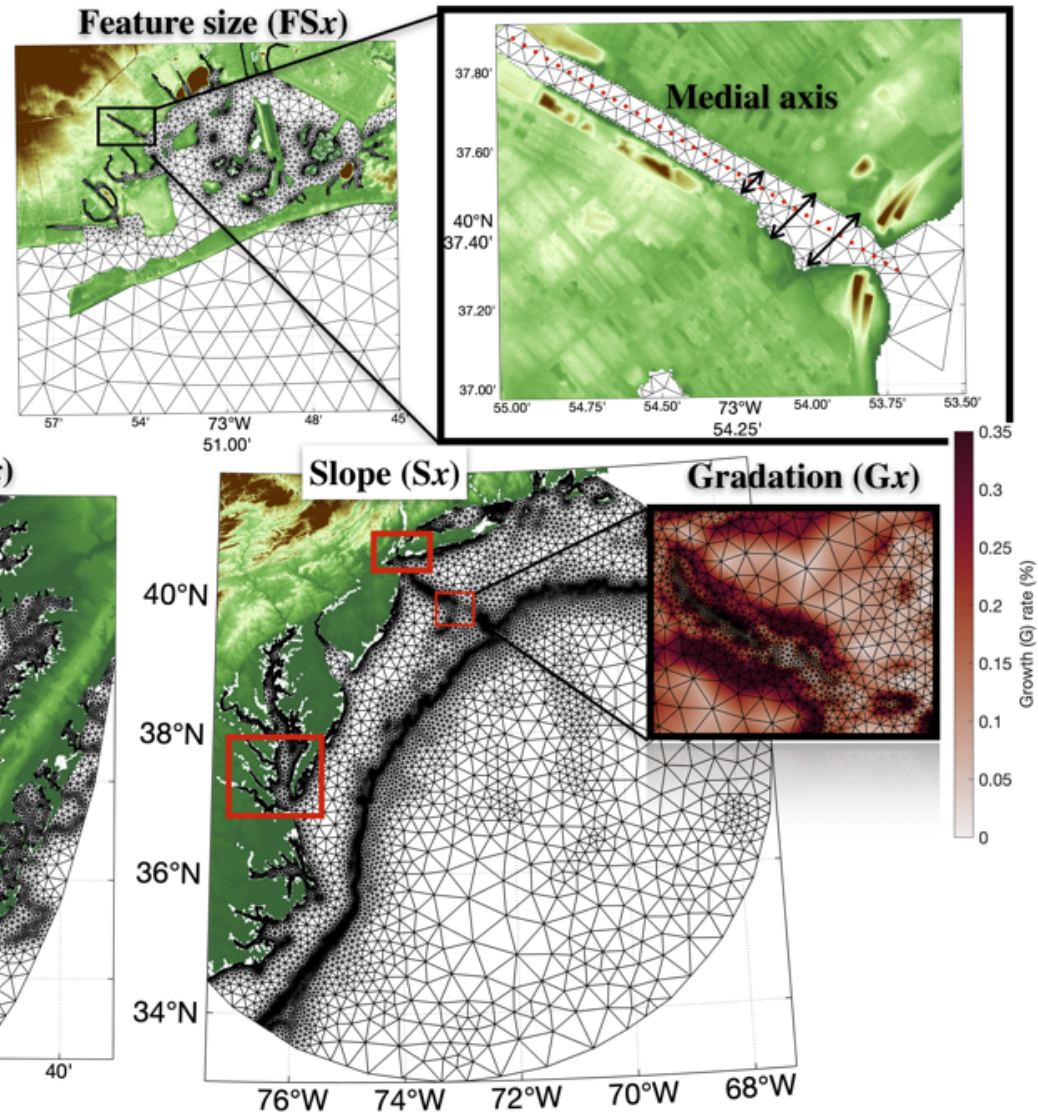

Figure 2. An illustration of the five mesh size functions that were investigated in and around the Mid-Atlantic Bight region along the Eastern United States coastline. Seabed topography is colored and relevant quantities are noted in the text. 
Table 1. The five experiments explored each containing three meshes in which the variable mesh size function parameter is indicated by $x$. The properties of the finely-resolved REF mesh used for a baseline comparison is also shown.

\begin{tabular}{|c|c|c|c|c|c|c|c|}
\hline Experiments & Fine & $\begin{array}{l}\text { Meshes } \\
\text { Medium }\end{array}$ & Coarse & $\begin{array}{l}\text { Mesh Size Function } \\
{[\mathrm{m}]}\end{array}$ & $L_{\min }[\mathrm{m}]$ & $L_{\max }[\mathrm{km}]$ & $g[\%]$ \\
\hline 1: Minimum Mesh Size & L50 & L150 & $\mathrm{L} 250$ & $\mathrm{~L} x=x+0.15 d_{s}$ & $\mathrm{x}$ & 10 & 15 \\
\hline 2: Feature Size & FS8 & FS4 & $\mathrm{FS} 2$ & $\mathrm{FS} x=2 * \frac{\left(d_{s}+d_{m}\right)}{x}$ & 50 & 10 & 15 \\
\hline 3: Grade & G15 & G25 & G35 & $\mathrm{G} x \Rightarrow 100\left|\frac{L_{i}-L_{j}}{X_{i}-X_{j}}\right|<x$ & 50 & 10 & $\mathrm{x}$ \\
\hline 4: Slope & S20 & S10 & S5 & $\mathrm{S} x=\frac{2 \pi}{x} \frac{b}{|\nabla b|}$ & 50 & 10 & 15 \\
\hline 5: Submarine channels & C1.0 & $\mathrm{C} 0.5$ & $\mathrm{C} 0.1$ & $\mathrm{C} x=\frac{2 \pi}{x} \frac{b}{\tan \left(30^{\circ}\right)}$ & 50 & 10 & 35 \\
\hline Reference (REF) & - & - & - & $\mathrm{REF}=50+0.15 d_{s}$ & 50 & $\begin{array}{c}0.25: b<50 \mathrm{~m} \\
1: b<250 \mathrm{~m} \\
5: b>250 \mathrm{~m}\end{array}$ & 15 \\
\hline
\end{tabular}

$L_{\text {min }}:$ minimum mesh size

$L_{\text {max }}$ : maximum mesh size

$L_{i}$ : mesh size at $i$ defined by the circumradius of each triangle

$X_{i}$ : coordinate of $i$ on grid to compute edgelengths

$i$ and $j$ : adjacent elements

$g$ : gradation

$d_{s}$ : shortest distance to the shoreline

$d_{m}$ : shortest distance to the medial axis

$b$ : topo-bathymetric depth (positive below sea level)

$\nabla$ : gradient operator

tide $\left(\mathrm{M}_{2}\right)$ since this is the predominant tidal constituent along the ECGC. The major diurnal tide $\left(\mathrm{K}_{1}\right)$ is also included where relevant.

The tidal elevations are decomposed into harmonic constituents using a least-squares method at all points within the domain. Relative errors (RE) in harmonic tidal elevation amplitudes from all sequences of experiments are calculated by linearly interpolating the solution from the REF mesh onto the experiment under consideration, subtracting the solutions, and then normalizing by REF, i.e.,

$$
R E=\frac{A_{I D}-A_{R E F}}{A_{R E F}} \times 100[\%]
$$

where $A$ is the harmonic elevation amplitude of the tidal constituent in the experiment (ID) and the REF meshes. A focus is placed on the $M_{2}$ and $K_{1}$ elevation amplitudes as these represent the predominant semi-diurnal and diurnal constituents in the ECGC domain (Section 2.1).

The calculation of the RE is proceeded in this manner to keep data extrapolation to a minimum so that the same shoreline geometric complexity as depicted in each mesh is present in both solutions under comparison. For all differences, statistics are only performed on vertices in which the absolute difference from REF exceeds $1 \mathrm{~mm}$ or the RE between solutions is greater than $0.1 \%$. These significance values are considered sufficiently small to ignore for the modeling purposes of barotropic tides along the ECGC, which have magnitudes on the order of centimeters to meters.

The convergence characteristics of the experiments are examined by comparing the cumulative area fraction errors (CAFE) of the RE statistic along the continental shelf margins of the ECGC region $(b<250 \mathrm{~m})$ where high mesh resolution zones were deployed (i.e., union of the green and red colored zones in Figure 1). To be consistent throughout, CAFE curves only consider errors $\left(A_{I D}-A_{R E F}\right)$ that exceed $1 \mathrm{~mm}$ or feature a RE greater than $0.1 \%$. On these CAFE plots, 
the y-axis value of a point falling on these curves indicates the percent area having a difference greater (less) than the positive (negative) value on the x-axis. A solution that has "converged" indicates that $99 \%$ of the comparison region has a $\pm 5 \%$ RE. This definition of convergence may be arbitrary but it represents a statistic that can enable a consistent comparison between solutions and a more strigent accuracy standard than currently set by the U.S. COASTAL Act ${ }^{1}$.

Last, in Section 3.5 we summarize the experiments through the standard deviation of the variation in the RE statistics from the REF mesh. Further, the contribution of numerical error versus error in the physical approximation of the domain is illustrated. Finally, based on the results of the five experiments described above we generate mesh designs that combine mesh size functions/experiments together to create a mesh with fewer vertices that can approximately mimic the tidal solution accuracy of the REF mesh.

The following set of statistics are computed to compare the accuracy, in terms of error against tide gauge observations (Section 2.1.2), of the simulated tidal solutions between the REF mesh and the combination mesh designs.

$$
\begin{gathered}
E=\left(0.5\left[A_{o}^{2}+A_{m}^{2}-2 A_{o} A_{m} \cos \left(\theta_{o}-\theta_{m}\right)\right]\right)^{1 / 2} \\
B=\frac{\sum_{t=1}^{T}\left(E_{I D}-E_{R E F}\right)}{\sum_{t=1}^{T} E_{R E F}} \\
\gamma^{2}=\frac{\operatorname{var}\left(E_{I D}-E_{R E F}\right)}{\operatorname{var}\left(E_{R E F}\right)}
\end{gathered}
$$

where $E$ is the complex root-mean-square error of a tidal constituent for one cycle and account for the amplitude and phase errors, $A$ and $\theta$ are the amplitudes and phase lags of the tidal constituent respectively, the subscripts 'o' and ' $m$ ' refer to the observed and modeled values respectively, and $T$ in the sum is the total number of tide gauges. $B$ is the normalized mean bias and $\gamma^{2}$ is the normalized variance (var) of the discrepancies of $E$ between the REF mesh and a particular mesh combination (ID). A positive value of $B$ indicates that the mesh combination has on average greater values of $E$ than REF, while a negative bias indicates the model is outperforming the REF solution. The smaller the value of $\gamma^{2}$, the more similar the mesh's solution is to REF in terms of the distribution of $E$. Since, a model can be tuned to fit observations locally, such as by employing variable bottom friction coefficients in regions where errors arise, the main aim here is to minimize $\gamma^{2}$ and $B$, through the effects of mesh resolution on the solution under the assumption that REF is sufficiently resolved. For reference, the REF solution has a median $E$ for the $\mathrm{M}_{2}$ of $3.9 \mathrm{~cm}$ (computed on all 667 tide gauges, Section 2.1.2).

\section{Results}

\subsection{Resolving the shoreline}

The representation of the shoreline determines the simulated accuracy in modeling the physical interaction between forcing agents (e.g., tides, winds, and waves) with shoreline geometrical features (e.g., coves, headlands, back-bays, and lagoons). From a modeling standpoint, the shoreline's representation must be simplified to satisfy computational resources by removing fineshoreline details from the mesh's boundary description that are smaller than the minimum mesh resolution. However, when the shoreline is simplified, it alters the approximation of the physical domain, and hence possibly the system's tidal response [e.g., Molines et al., 1989; Greenberg et al., 2007].

${ }^{1}$ https://www.weather.gov/sti/coastalact 

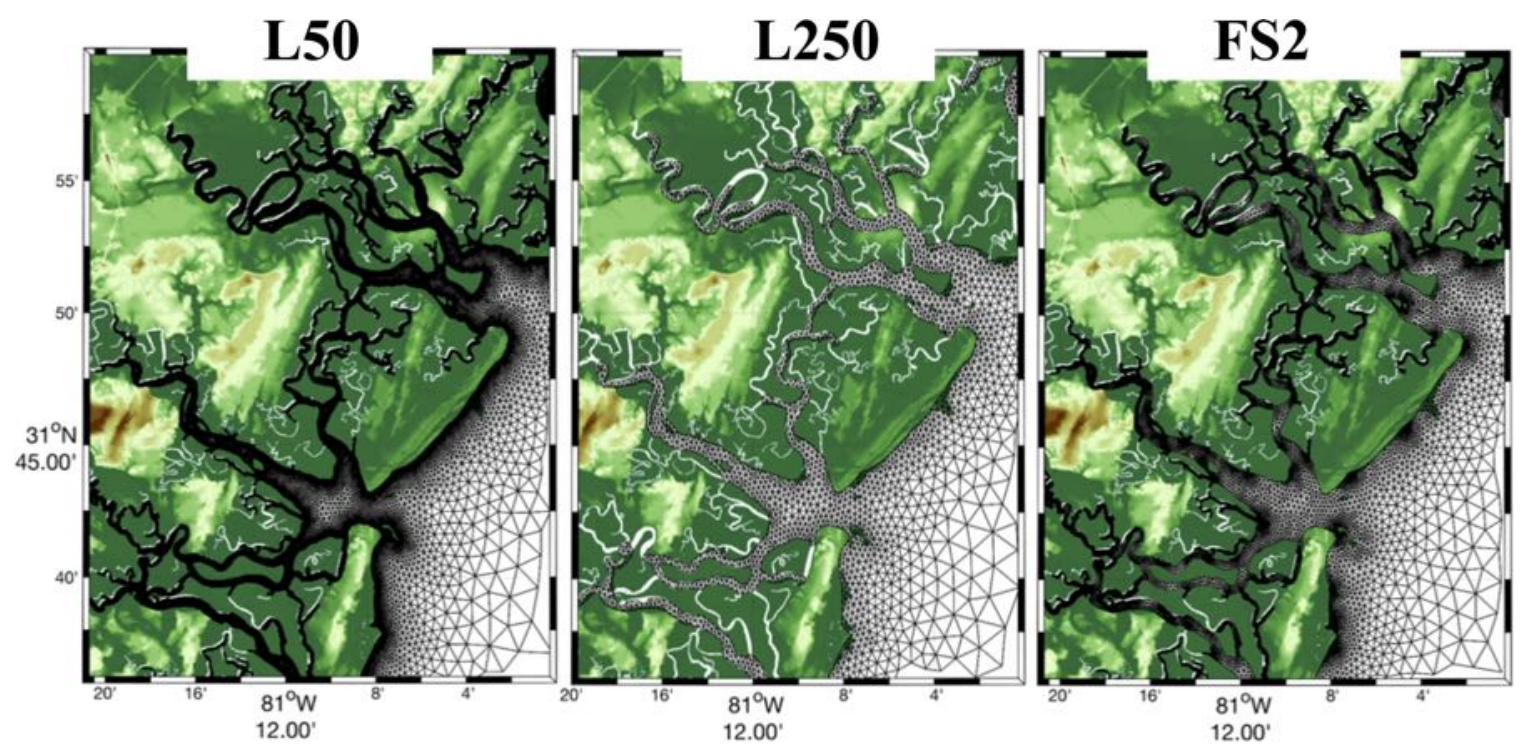

Figure 3. Mesh connectivity near Ossabaw Island, Georgia that illustrates changes to the capturing of narrow channel geometries as minimum mesh resolution is increased from $50 \mathrm{~m}$ (left) to $250 \mathrm{~m}$ (middle), and when using a shoreline width function that varies minimum mesh resolution between $50 \mathrm{~m}$ and $250 \mathrm{~m}$ (right) automatically based on shoreline geometric properties.

This section uses the results from Experiments $1(\mathrm{~L} x)$ and 2 (FS $x)$ to explore the effects of varying a specified minimum resolution at the shoreline and of varying shoreline resolution according to a feature size estimation, respectively. A comparative example of the L $x$ and FS $x$ designs along an estuarine region is illustrated in Figure 3. As the minimum mesh resolution is coarsened from $50 \mathrm{~m}$ to $250 \mathrm{~m}$, narrow waterways, tributaries, and estuaries that are smaller in horizontal length-scale than the minimum mesh resolution are automatically removed in the mesh generation process [Roberts et al., 2018]. The removal of fine-scale shoreline geometry is considered a shoreline approximation error in the sense that the approximate representation of the shoreline departs from its representation in the original shoreline dataset. In contrast, the feature size approach creates a mesh that represents the physical system accurately by connecting small waterways together in a similar manner to L50, but requiring fewer vertices as resolution can expand in size away from geometric constrictions along the shoreline (Figure 3).

It is important to note that the variation in the minimum element size along the shoreline will impact the sizing of elements near and along adjacent inner and outer shelf seabed topographical features as all the meshes are graded to expand in element size offshore. In addition, the application of the FS $x$ will lead to finer resolution near more irregular shoreline features. Considering this, more pronounced differences in element sizes will tend to occur between FS $x$ and L $x$ in proximity to shoreline segments that are highly irregular in their form. Thus, besides the obvious impact on the representation of the shoreline via either the Lx or FS $x$ design, the variations in this experiment also implicitly alter the representation of the inner and outer shelf seabed topographic gradients.

The shoreline approximation error is quantified by integrating the area enclosed by the polygonal region that defines the mesh boundary ( $\mathcal{S}$ in which the sub-script denotes the experiment ID).

$$
\mathcal{A}_{\text {error }}=\left|\mathcal{S}_{I D}-\mathcal{S}_{\text {Ref }}\right|
$$




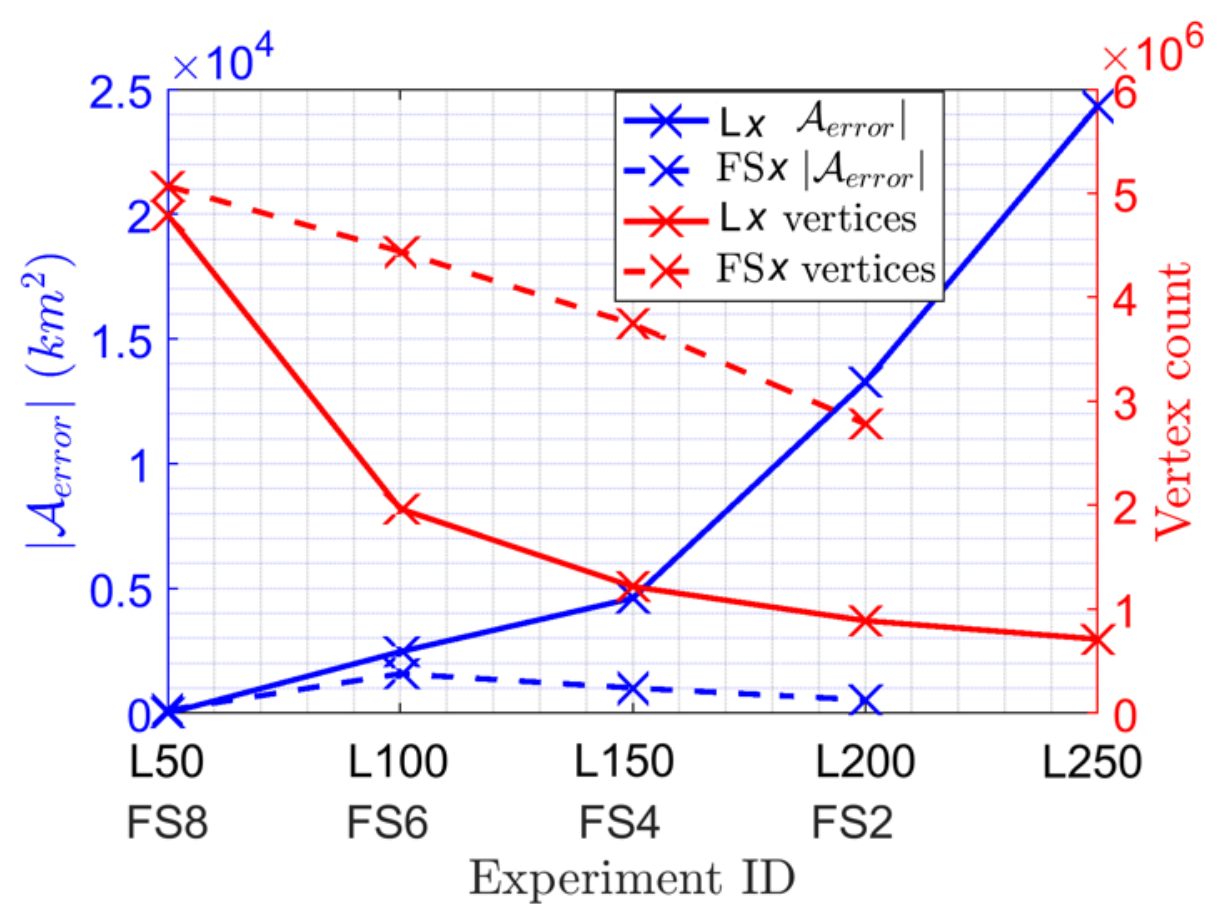

Figure 4. The shoreline geometry error $\mathcal{A}_{\text {error }}$, Equation (5), on the left axis for the meshes used in the shoreline approximation experiment along with the total vertex count for each mesh on the right axis. Solid lines represent data for meshes created with uniform shoreline resolution $\mathrm{L} x$ and dashed lines indicate meshes created with the feature size approach FS $x$.

$\mathcal{A}_{\text {error }}$ increases geometrically as the minimum shoreline resolution is coarsened from $50 \mathrm{~m}$ to $250 \mathrm{~m}$ in the Lx meshes (Figure 4). For example, $\mathcal{A}_{\text {error }}=2,200 \mathrm{~km}^{2}$ for L100 increases approximately ten-fold to $\mathcal{A}_{\text {error }}=22,000 \mathrm{~km}^{2}$ for L250, while the total vertex count reduces from 4.9 million to 0.8 million vertices between L250 and L50 mesh designs. In contrast, the FS $x$ experiments exhibits no correspondence between total vertex counts and shoreline approximation error and $\mathcal{A}_{\text {error }}$ remains small reaching a maximum of approximately $1,500 \mathrm{~km}^{2}$. The FS $x$ design distributes 50-m mesh sizes in narrow waterways and along high curvature shoreline sections, while allowing mesh sizes to expand up to $250 \mathrm{~m}$ along straighter shoreline segments. The predominate variation in vertex counts in the FS $x$ design is the number of vertices per geometric width of the shoreline, not the minimum element size. Thus, the FS2 design is capable of preserving a similar amount of shoreline geometry as L50 (e.g., Figure 3a,c) but with approximately two times fewer vertices.

As is evident in Figure 5, the variation in the representation of the shoreline predominately affects the $\mathrm{M}_{2}$ elevation amplitude in shallow shelf regions ( $<250$-m depth range). A largely insignificant error $(<1 \mathrm{~mm}$ or $\pm 0.1 \%)$ was observed in the $\mathrm{K}_{1}$ elevation amplitude (not shown). The relative $\mathrm{M}_{2}$ errors (RE) among the $\mathrm{L} x$ experiments are greatest for L250 and smallest for L50 (Figure 5a-b), demonstrating the improvement of finer resolution. Large RE values are concentrated in estauries in the $\mathrm{SAB}$ and in the MAB around the Chesapeake Bay and the Gulf of Maine where large RE values of 10-15\% are found in the L250 mesh (Figure 5b). In the MAB, SAB, and eastern GOM shelf zones, there is a weak 1-3\% deamplification in the $\mathrm{M}_{2}$ amplitude with the exception of the Chesapeake Bay estuary, which exhibits a pronounced RE of $+5-10 \%$ as the mesh resolution is coarsened from L50 to L250. In general, the FS $x$ meshes (Figure 5c-d) produce similar relative error patterns to the $\mathrm{L} x$ meshes. However, negative RE values are only $<1 \%$ in the Chesapeake and SAB for the coarsest Lx design (FS2) compared to RE values in L250 which are 


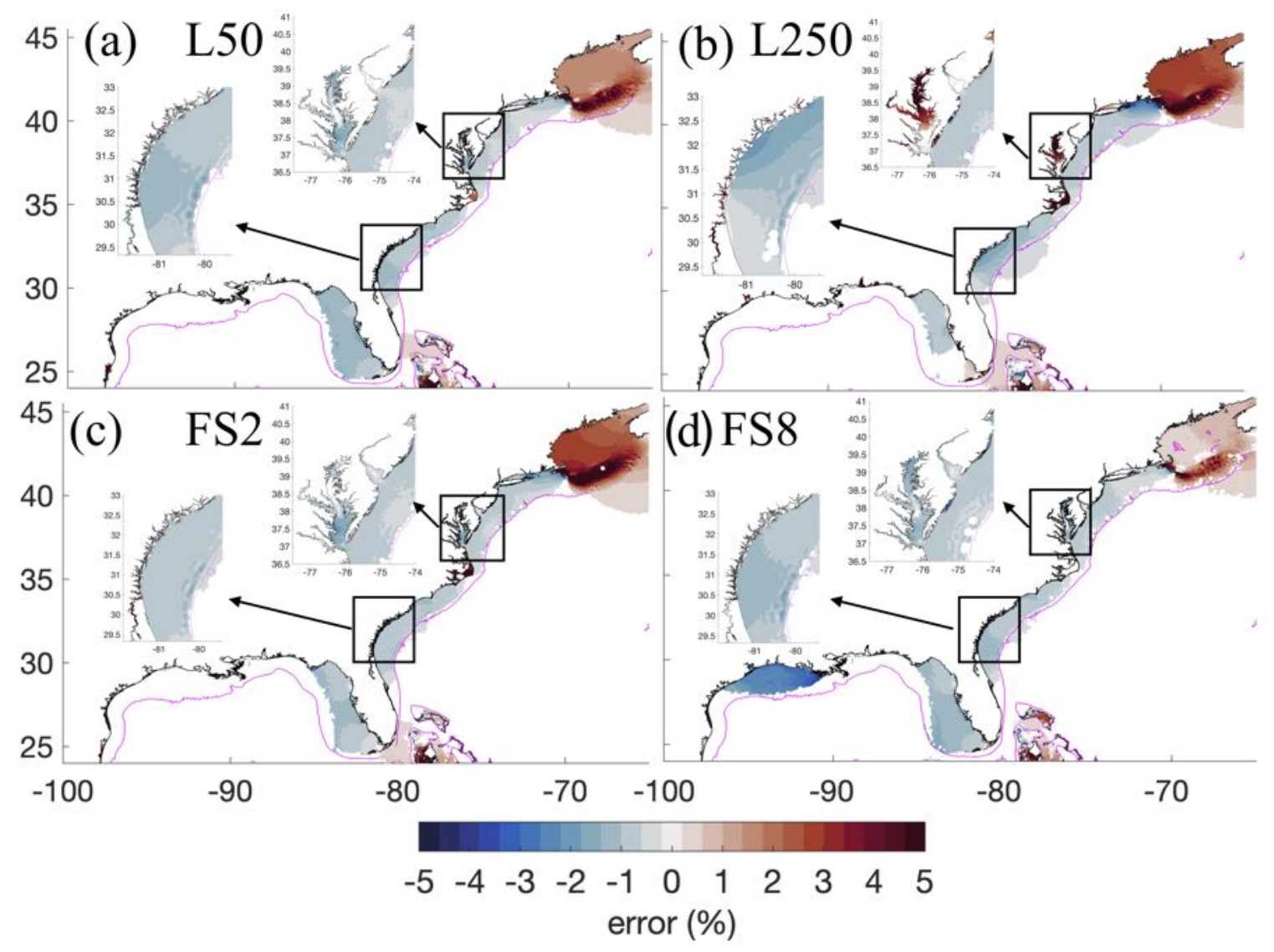

Figure 5. Panels (a)-(b) depict the relative error in the $\mathrm{M}_{2}$ harmonic elevation amplitude from the REF solution when the minimum mesh resolution along the shoreline is coarsened from $50 \mathrm{~m}$ and $250 \mathrm{~m}$. Panels (c)-(d) depicts the relative error (RE) in the $\mathrm{M}_{2}$ harmonic elevation amplitude from solutions computed on meshes built with the feature size function. Insets around areas described in more detail are shown. 

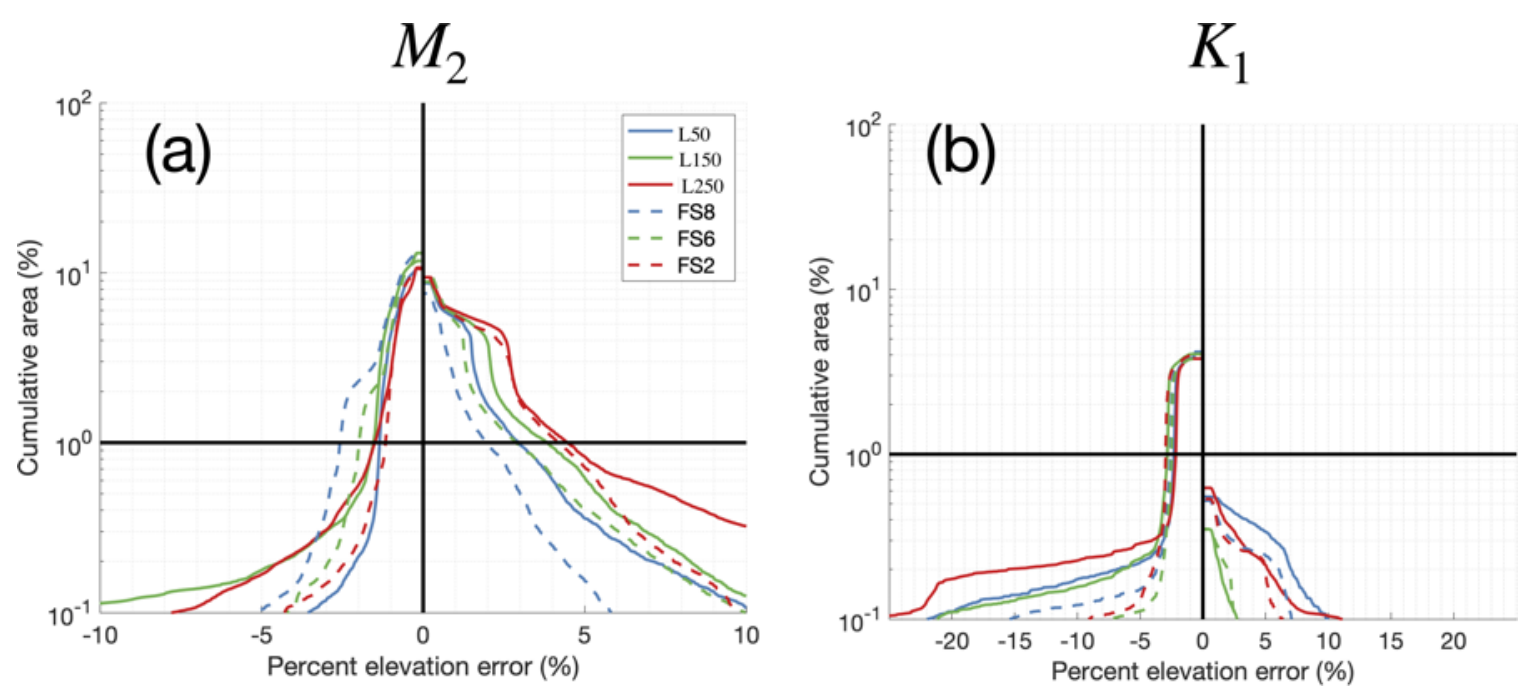

Figure 6. The cumulative area fraction error (CAFE) from the REF solution in the comparison region for panel (a) the $\mathrm{M}_{2}$ elevation amplitude and panel (b) the $\mathrm{K}_{1}$ elevation amplitude. The dotted lines denotes solutions computed on meshes that use the FS $x$ design while the solid-lines denote meshes created with the L $x$ design.

approximately $\pm 3 \%$ here. Further, FS2 reduces the amplification in the Gulf of Maine by a small amount $\sim 1 \%$. The western GOM shelf region weakly deamplified by $1-3 \%$ in the FS8 design, but this was not observed in the other L $x$ designs.

Although local differences in RE are illustrated in Figure 5, the CAFE curves demonstrate remarkable similarity in $99 \%$ of the comparison zone between the Lx and FS $x$ solutions (i.e., above the thick $1 \%$ cumulative area line) for both the $\mathrm{M}_{2}$ and $\mathrm{K}_{1}$ elevation amplitudes (Figure 6). The CAFE curves for the $\mathrm{M}_{2}$ are asymmetrical and indicate more of the domain has a positive error, which is accentuated in the tails below the $1 \%$ cumulative area line. While all the solutions in this experiment have achieved a converged solution, the FS6 and FS8 contain less positive RE than the $\mathrm{L} x$ designs, while the opposite is true for the negative crossing although the difference is marginal $(1-2 \%)$.

The relatively coarser L250 (+4.0\% RE) and FS2 (+3.9\% RE) mesh designs exhibited only slightly larger positive errors in the $\mathrm{M}_{2}$ elevation amplitude as compared to L50 and the FS8 design. These differences are marginal considering the 4 million total vertex count difference between the fine and coarse mesh designs (i.e., L50 and FS8 vs. L250). For the $\mathrm{K}_{1}$, all meshes have converged solutions to our tolerance and respond far less to alterations in mesh design than the $\mathbf{M}_{2}$.

\subsection{Mesh size gradation}

The concept of grading is a key capability of unstructured mesh finite element or finite volume modeling in which coarse elements in the far-field grade smoothly into the more finely resolved region of interests where fine resolution is necessary to capture the physical system and/or the hydrodynamic response to efficiently discretize regional and global ocean domains. This gradation rate between zones of variable resolution can greatly influence the number of vertices in the mesh (Figure 7). Elemental size grading has been based on solution gradients as well as bounding an estimate of the Courant number to encourage numerical stability [Luettich and Westerink, 1995]; however, the grade can also be based on geometric criteria by ensuring that neighboring mesh element sizes cannot enlarge too quickly [Persson, 2006], i.e., the gradation is a bound on the maximum relative incease in edgelength between adjacent elements. It is understood from a general modeling point of view that excessive gradation rates lead to triangles with skewed triangles containing acute or obtuse angles, which can impact the stability and numerical accuracy of the model 
[Massey, 2015; Shewchuk, 2002]. Further, the analysis by Hagen et al. [2000] for one dimensional domains demonstrates that a high gradation value $(g \approx 0.5)$ leads to the introduction of odd order truncation error term, which lowers the order of the method to first-order accurate and/or degrades the local/global accuracy.

Fundamentally, the gradation rate will impact many aspects of the mesh design at once. A higher valued mesh size gradation will degrade the approximate representation of the seabed topography by creating comparatively coarser mesh sizes away from the targeted zones of fine resolution. As was described in Table 1, the meshes that vary the gradation rate utilize a minimum resolution of 50-m along the shoreline (L50). Note that the mesh generator is bounding the gradation rate above by the user-defined parameter value only on the mesh size function and it is assumed that given the convergence of the mesh generator the gradation rate is similarly bounded in the triangulation (Section 2.4). Coarser mesh sizes tend to smooth the interpolation of seabed features onto the mesh vertices and this data interpolation effect can be quantified in the meshes by calculating the overall volume enclosed by the mesh while holding the shoreline boundary fixed (i.e., the surface area of the total mesh is constant). Thus, similar to the shoreline approximation error (Eq. 5), the seabed approximation error is calculated as the absolute difference in total volume from the REF mesh:

$$
\mathcal{V}_{\text {error }}=\left|V_{I D}-V_{R E F}\right|
$$

where $V$ is the total mesh volume for the mesh denoted by $I D$ and is calculated as the sum of all the mesh element volumes. An element volume is calculated by multiplying the average depth of the element by its area. Since the REF mesh employs uniform high resolution mesh sizes throughout the nearshore and continental shelf zones (c.f., Figure 1), it represents the seabed surface with the smallest approximation error. Note that the data interpolation approach we are using is a gridscale average (Section 2.3) and is not a globally conservative interpolation scheme. From Figure 7, it is apparent that there is a diminishing reduction in the total vertex count of the mesh with increased gradation. For the purposes of this study, we were not able to explore meshes with gradation greater than 35\% due in Experiment $3(\mathrm{G} x)$ due to the introduction of triangles with very skewed aspect ratios and obtuse and acute angles that created numerical accuracy issues.

The increase in mesh size gradation from $15 \%$ to $35 \%$ leads to a highly amplified error pattern in the NA region for both $\mathrm{M}_{2}$ and $\mathrm{K}_{1}$ constituents as well as along the MAB for $\mathrm{M}_{2}$ (Figure 8). In the NA subdomain (Gulf of Maine), the $\mathrm{M}_{2} \mathrm{RE}$ is increased from 2-5\% for G15 to 10$21 \%$ for G35 (colors are saturated in Figure 8a), in which the maximum RE is focused on the Georges Bank. In contrast to the response in the $M_{2}$ 's RE, the $\mathrm{K}_{1}$ 's RE is nearly uniformly degraded from $-3 \%$ for G15 to $-6 \%$ for G35 in the NA subdomain. The $\mathrm{M}_{2}$ RE in the MAB, SAB, and eastern GOM tends to weakly deamplify by approximately $1 \%$ to $5 \%$ along the continental shelf zones. In contrast to the shoreline approximation experiment, a relatively large deamplification of the $\mathrm{M}_{2} \mathrm{RE}$ occurs in both the Chesapeake Bay and Delaware Bay as the gradation is enlarged to $35 \%$ (Figure 8a,b). The $\mathrm{M}_{2} \mathrm{RE}$ reaches as high as 15\% in this region for the G35 experiment (colors are saturated in Figure 8a).

As the mesh size gradation grows, the tidal elevation amplitudes start to diverge substantially from the REF solution (Figure 9). In 99\% of the comparison zone, the G15 mesh has an $\mathrm{M}_{2}$ error between $-1.3 \%$ and $+3.0 \%$ RE whereas G35 has between $-5.0 \%$ and $+15 \%$ RE. Furthermore, the G35 mesh design exhibits between $-5.5 \%$ and $+6.5 \% \mathrm{~K}_{1} \mathrm{RE}$ in the $99 \%$ comparison zone, which is compared to $-3.0 \%$ and $+0 \% \mathrm{~K}_{1} \mathrm{RE}$ for G15. Unlike the shoreline experiment where all meshes converged (based on the $\pm 5 \%$ threshold definition of convergence), only the G15 mesh converges for the $\mathrm{M}_{2}$ constituent, and the G15 and G25 meshes converges for $\mathrm{K}_{1}$. However, it's important to note that the tendency of the solution is convergent as the RE reduces when the mesh sizes are made finer with lower gradation bounds. 


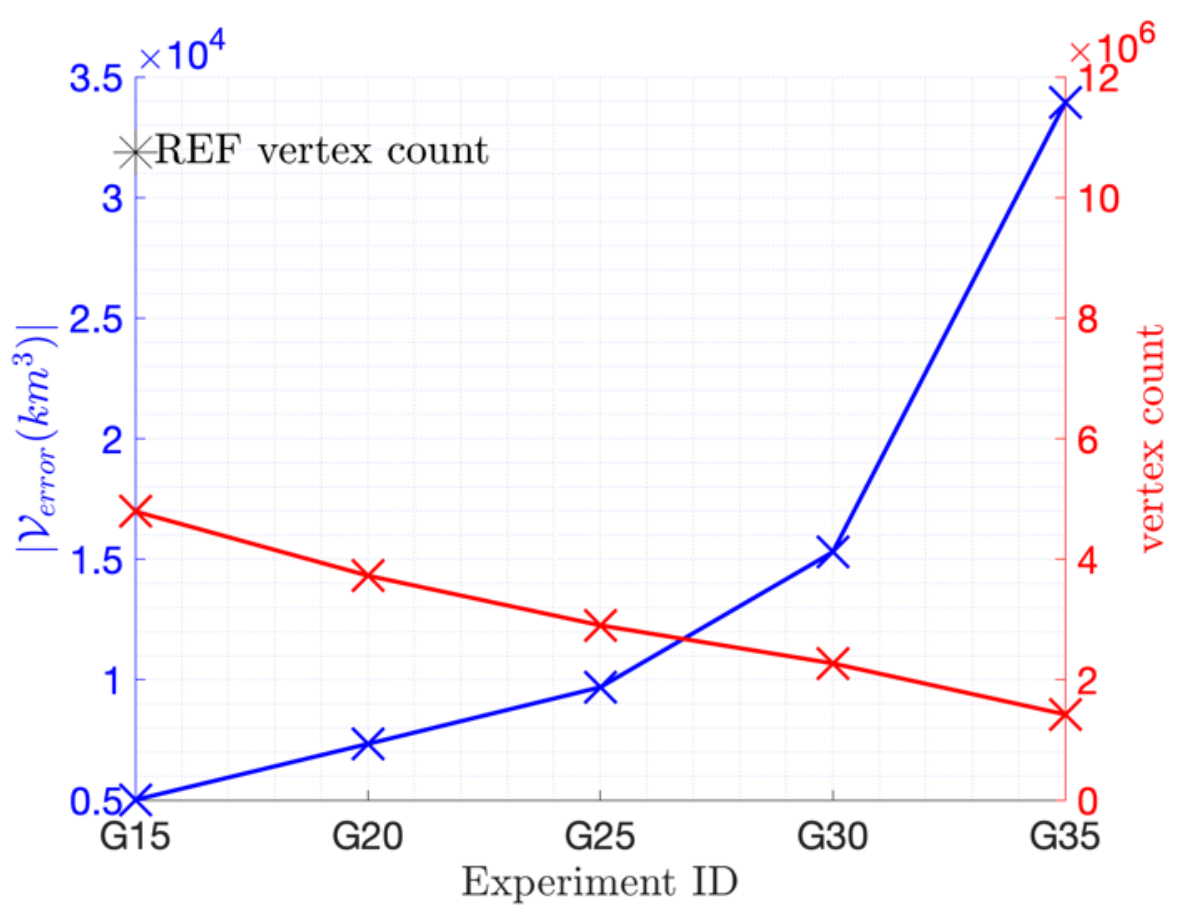

Figure 7. The seabed approximation error $\mathcal{V}_{\text {error }}$ (Equation 6) on the left-axis (blue x's) as the mesh size gradation is increased from $15 \%$ to $35 \%$ in increments of $5 \%$ while the shoreline boundary is held fixed (i.e., area of domain is constant). The total vertex count in each mesh on the right axis (dashed red x's). The REF mesh vertex count is demarcated by a black asterisk in the top left corner of the figure.

\subsection{Resolution along bathymetric gradients}

The main motivation for increasing the horizontal resolution in the open ocean is to more accurately represent sharp seabed gradients, particularly those that characterize the continental shelf break and slope. The representation of these seabed gradients is captured with the topographiclength-scale Sx (Figure 2 and Table 1). The topographic-length-scale $\mathrm{S} x$ is considered a useful mesh heuristic [see Greenberg et al., 2007, for a review] to aid in the modeling of shelf break dynamics [Huthnance, 1995; Hannah and Wright, 1995; Luettich and Westerink, 1995], subtidal dynamics [Loder, 1980; Chen et al., 2016], and internal tide generation processes [Xing and Davies, 1998] and their effects on barotropic tides [Pringle et al., 2018a,b]. Further, Hagen et al. [2001] has demonstrated that an inadequate prescription of resolution along sharp seabed gradients is a source of numerical truncation error for tidal models. However, as $b \rightarrow 0$, the Sx meshing criteria fails for some areas as resolution becomes excessively fine in shallow depths and creates element sizes which can lead to numerical instabilities.

The topographic-length-scale $\mathrm{S} x$ parameter must consider the trade-off between the improvement to the solution of barotropic tides and the additional mesh vertex count. Chen et al. [2016] suggested resolution sizes between 3.3 to $6 \mathrm{~km}$ to capture the shelf break and $2 \mathrm{~km}$ to capture the deep slope in the Arctic Ocean. Lyard et al. [2006] suggested S15 globally using quadratic finite elements, but noted that this value was restricted in its spatial application due to the excessive computational expense it incurred. In our studies, besides the excessive computational expense incurred by the additional degrees-of-freedom, we have found that using Sx larger than S20 leads to resolution along the shelf-break that can extensively restrict the feasible time step (i.e., time step of $2 \mathrm{~s}$ with Courant number bounded to 0.5). Note that the $\mathrm{S} x$ heuristic is only applied where $b>50$ $\mathrm{m}$ to avoid issues in shallow depths, where many small-scale features such as channels that we propose an alternative strategy to resolve documented later on. 


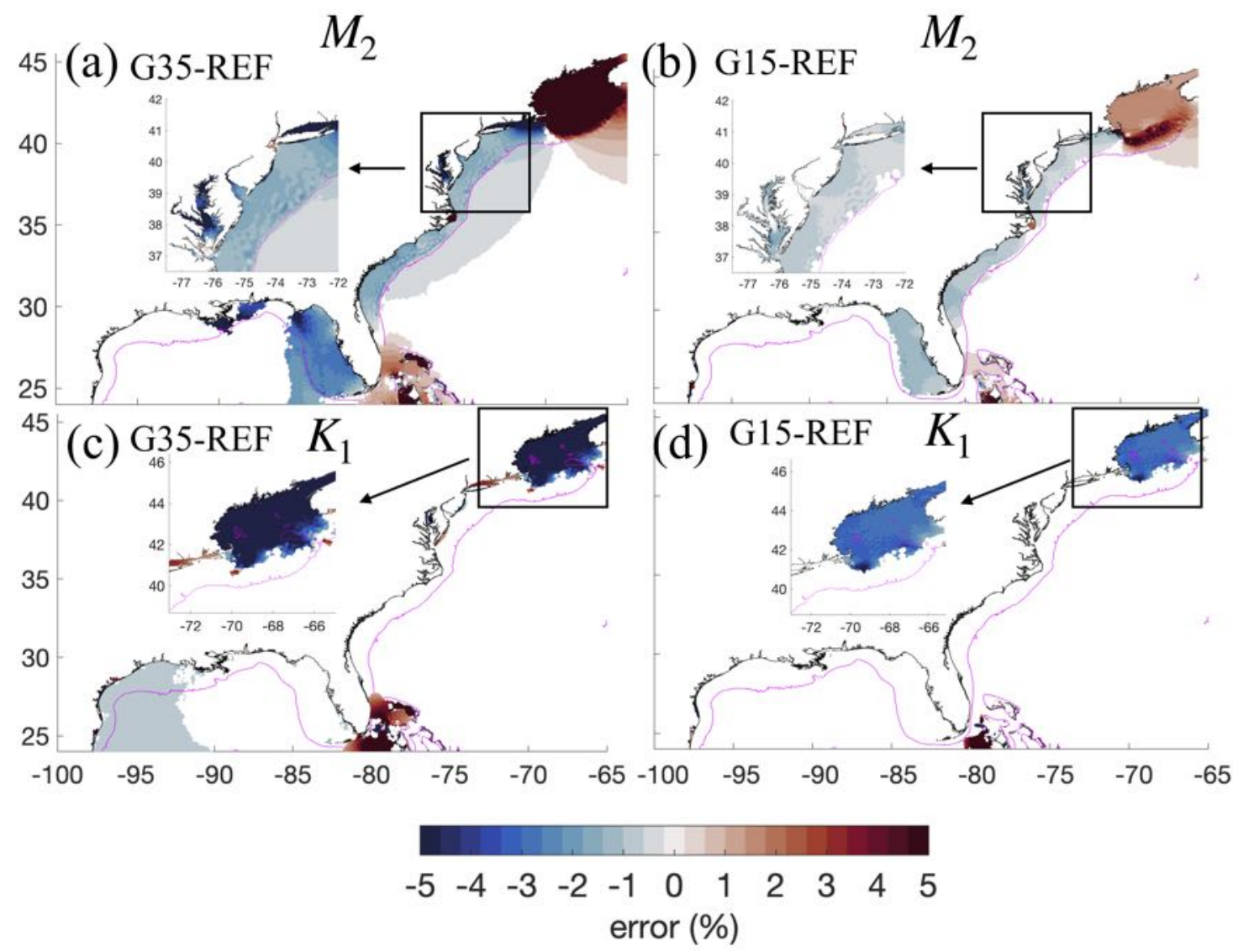

Figure 8. Panels (a)-(b) illustrate the RE in the $\mathrm{M}_{2}$ elevation amplitude from the REF solution as the mesh gradation bound is increased to $35 \%$ while in panel (b) it is kept low at $15 \%$. Panels (c) and (d) are the same as panels (a)-(b) but for the $\mathrm{K}_{1}$ elevation amplitude. The 250-m isobath contour is drawn as a magenta line in each panel for reference. Insets are shown to reflect areas that are described in the text.
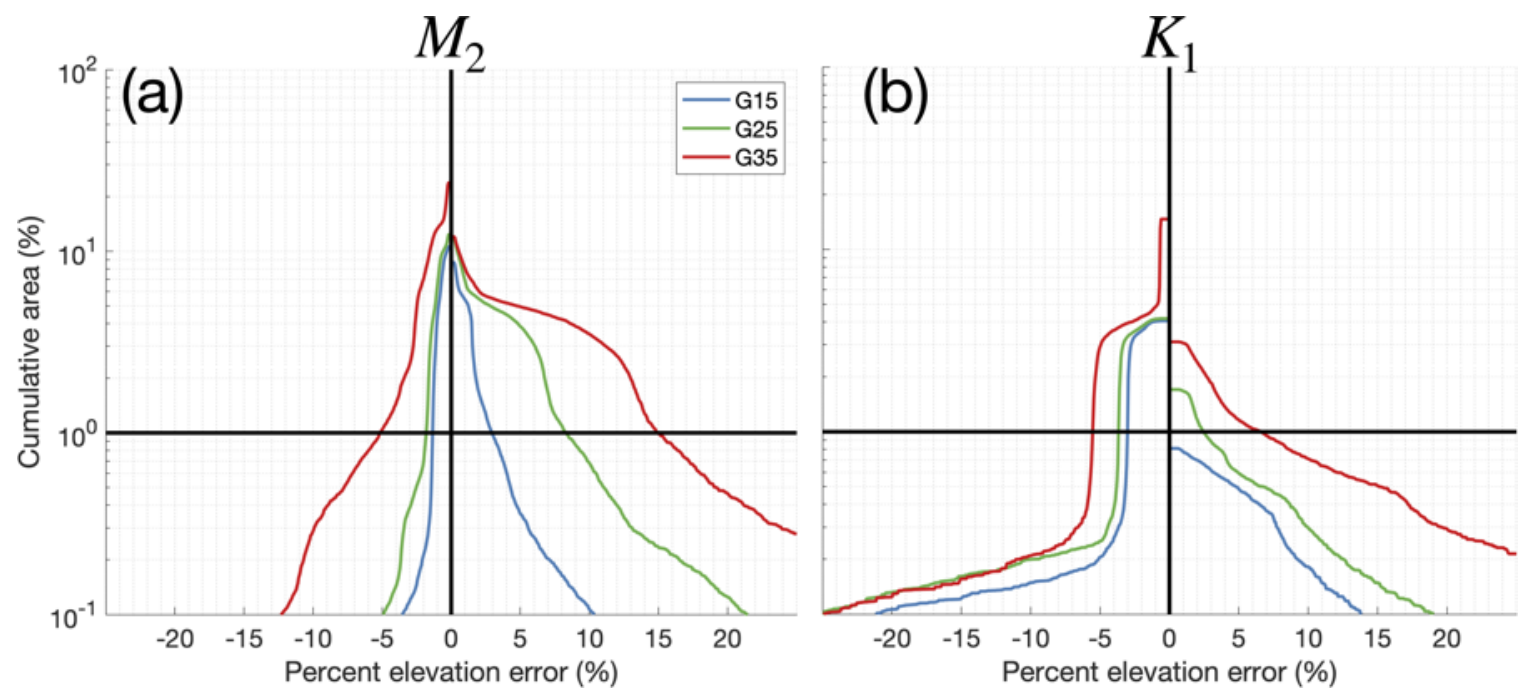

Figure 9. The cumulative area fraction error (CAFE) in the comparison zone (c.f., Figure 1) in panel (a) for the $\mathrm{M}_{2}$ elevation amplitude and panel (b) for the $\mathrm{K}_{1}$ harmonic elevation amplitude using the meshes created for the mesh size gradation experiment. 

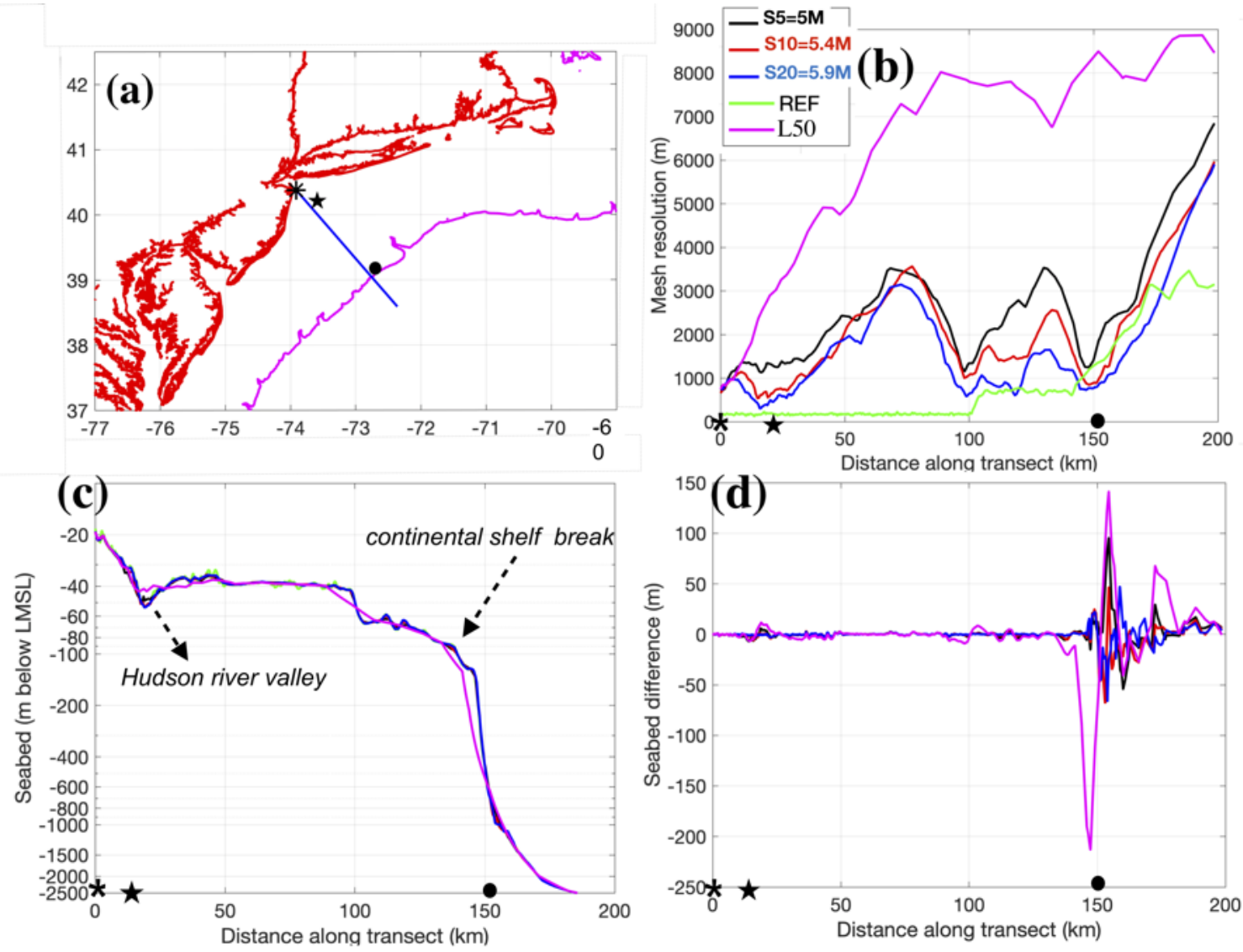

Figure 10. (a) A cross-shelf transect in the MAB region indicated in blue with the asterisk indicating the start of the transect, the magenta line is the $250-\mathrm{m}$ isobath, and the red line is the shoreline; (b) the mesh resolution along the transect for the Sx, REF, and L50 meshes. Panel (c) illustrates the seabed topography along the transect for each mesh. Panel (d) illustrates the difference in seabed topography from each mesh and the REF mesh along the transect. 

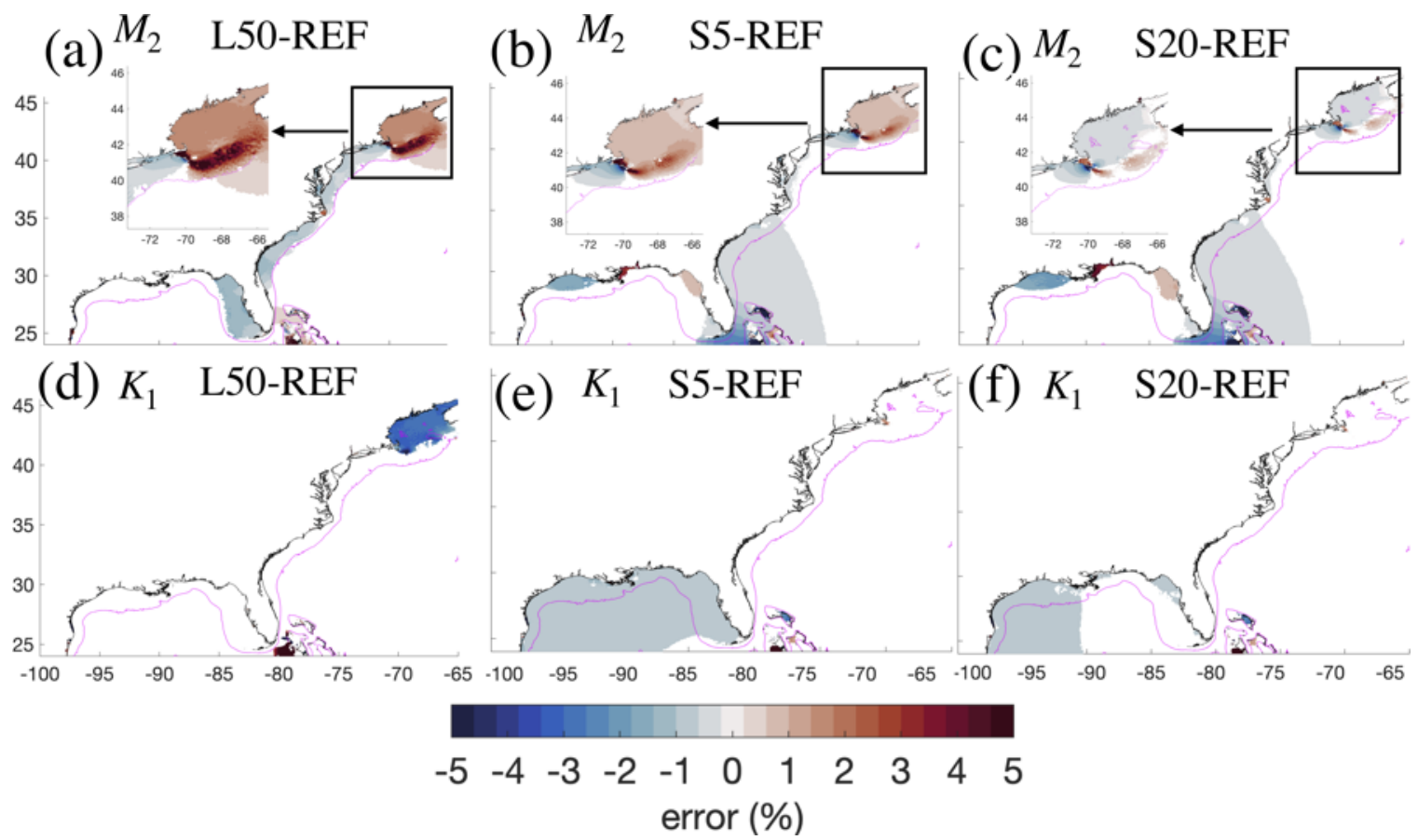

Figure 11. Panels (a)-(b) depict the $\mathrm{M}_{2}$ elevation amplitude RE for solutions computed on the $\mathrm{S} x$ meshes. Panels (c)-(d) depict the RE the $\mathrm{K}_{1}$ elevation amplitude.

In Experiment $4(\mathrm{~S} x)$ the vertex count is increased by $4 \%$ to $20 \%$ over the L50 mesh, accompanied by an improvement to the approximation of seabed profile, as illustrated along a transect spanning the cross-shelf direction in the MAB region (Figure 10). Mesh resolution in the vicinity of the shelf break zones is enhanced to approximately $1.2 \mathrm{~km}$ and $0.8 \mathrm{~km}$ for S5 and S20, respectively. A point worth noting is that seabed features exist on the continental shelf break, such as the drowned Hudson river valley, which will otherwise be completely smoothed over without the $\mathrm{S} x$ heuristic. In comparison, without $\mathrm{S} x$, resolution is coarser than $8 \mathrm{~km}$ (close to the maximum resolution size) in the vicinity of the shelf break (see L50 in Figure 10b), which tends to shift the break zone shoreward and result in a smoother and more gradual representation of the seabed profile along the transect (Figure 10c). The $S x$ heuristic results in a clear improvement in the depiction of the seabed profile. S20 had seabed profile differences of less than $50 \mathrm{~m}$ from the REF mesh, whereas the seabed profile difference for L50 is as large as $200 \mathrm{~m}$ (Figure 10d).

The finer resolution along seabed gradients using $\mathrm{S} x$ leads to a significant overall reduction in the RE pattern associated with the $\mathrm{M}_{2}$ elevation amplitude in the MAB and NA subdomains (Figure 11a-c), with the $\mathrm{M}_{2}$ error pattern diminished almost entirely for S20 (Figure 11c). Note that although the largest $\mathrm{RE}$ is co-located with the phase convergence zone of the $\mathrm{M}_{2}$ tidal species in the MAB and NA domain (where the elevation amplitude is zero), the RE is not confined to solely the amphidromic point and emanates around the entirety of the NA subdomain. Similarly, for the $\mathrm{K}_{1}$ elevation amplitude, an approximately $-4 \% \mathrm{RE}$ in the NA subdomain for the L50 (i.e., S0) mesh is undetectable for any of the Sx meshes (Figure 11d-f). Contrastingly, in the GOM domain the application of $\mathrm{S} x$ tends to introduce differences from the REF mesh rather than reduce them. Upon inspection, the REF is less resolved in parts of the GOM, Bahama Banks, and the Caribbean Sea (c.f., Figure 1) in comparison to the Sx meshes here, possibly explaining this result. The S20 mesh in particular contains finer resolution than the REF mesh along the shelf break zones of the western GOM, which is co-located with a persistent albeit weak negative RE in the $M_{2}$ in the S10 and S20 solutions. 

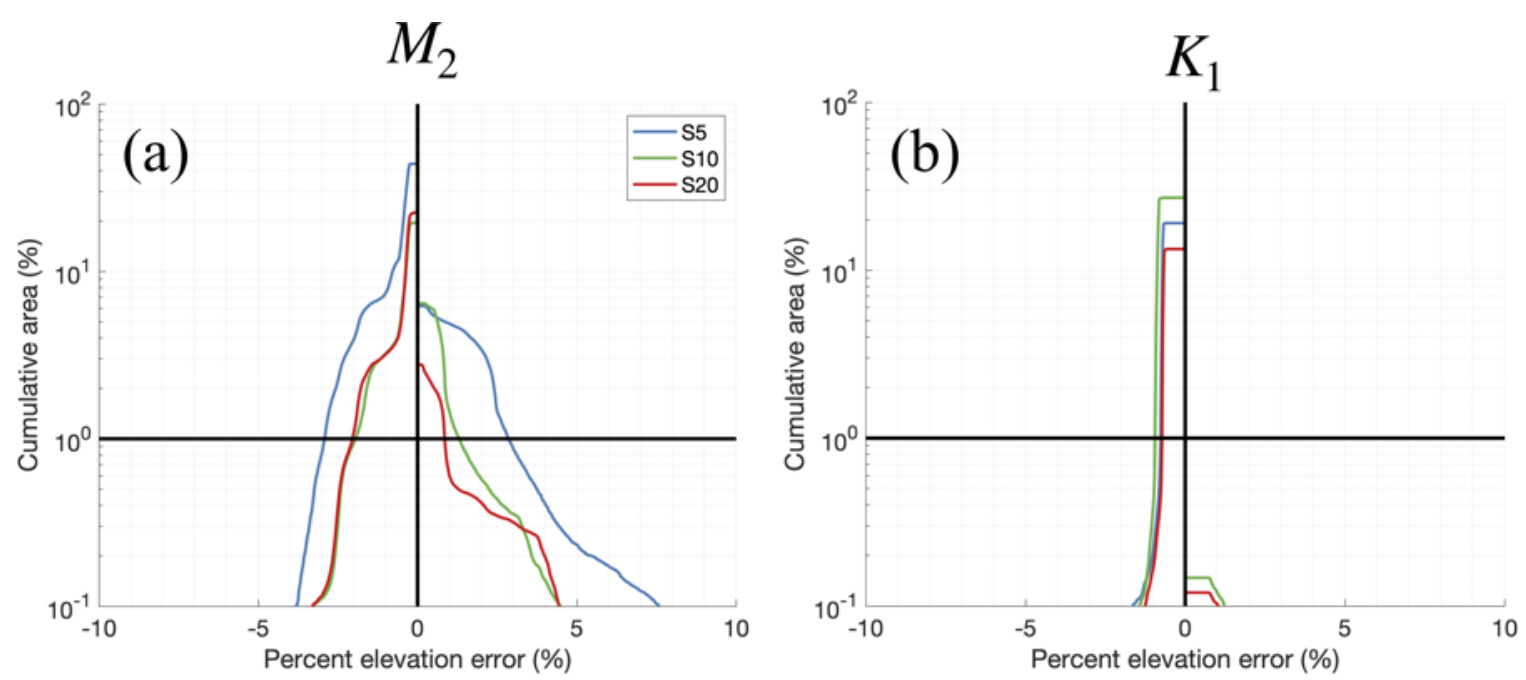

Figure 12. The cumulative area fraction error (CAFE) curves in the comparison zone for the Sx meshes.

The CAFE curves for $\mathrm{M}_{2}$ and $\mathrm{K}_{1}$ (Figure 12) clearly illustrate that increased resolution along seabed gradients leads to a converged solution in 99\% of the domain for S5, S10 and S20 according to our definition of convergence $( \pm 5 \%)$. The S5 mesh has the largest $\mathrm{M}_{2}$ error of $\pm 2.9 \%$ RE in $99 \%$ of the comparison zone, which predominantly corresponds to the errors in the MAB and NA domains. As evident from Figure 12, the $\mathrm{K}_{1}$ was less sensitive to the choice of $\mathrm{S} x$ mesh design than $\mathrm{M}_{2}$, with differences of approximately $\pm 3 \%$ in $99 \%$ of the comparison zone. However, the S $x$ for the $\mathrm{K}_{1}$ consistently and substantially (by 10 to $15 \%$ ) reduced the spread of the tails in $0.1 \%$ of the domain. As was illustrated in Figure 12(d),(e),(f), the negative underprediction for the $\mathrm{K}_{1}$ in the NA and MAB domains were consistently reduced with the application of the $\mathrm{S} x$ heuristic.

\subsection{Cross-sectional representation of estuarine channels}

Estuarine hydrodynamics are controlled by the depth and form, together referred to as the morphology of the estaurine seabed [Dronkers, 1986; Parker, 1991; Friedrichs, 2010; Prandle, 2003]. Thus, when designing a model to simulate coastal hydrodynamics, it is important to apply sufficient resolution to approximate the nearshore seabed topography. In particular, coarse mesh resolution in the presence of fine and narrow channelized bed forms will alias the channel's crosssectional profile (Figure 13) and lead to the inaccurate computation of transports, fluxes, and frictional resistance [Molines et al., 1989; Greenberg et al., 2007]. In the boarder context of mesh generation techniques for coastal ocean modeling, mesh design heuristics that target resolution inversely proportional to seabed's depth [e.g., Westerink et al., 2008] will also tend to coarsen the resolution in the center of the estuary in the deepest component of the tidal channel. Thus, exisiting techniques used to build models do not adequately resolve long and narrow channelized bed forms that are critical to conveying water into and throughout inland water systems.

An automatic mesh size function $\mathrm{C} x$ that localizes finer mesh resolution in close proximity to the thalwegs of important estuarine channel morphology was developed as part of the OceanMesh2D meshing software suite [Roberts et al., 2018]. An example of a mesh created with the estuarine channel mesh size function $\mathrm{C} x$ is illustrated in Figure 13(c) for the Delaware Bay estuary located in the MAB region. With $44 \%$ less vertices than REF in this subset of the ECGC, the C0.5 mesh represents the cross-sectional area of the deepest thalweg in the estuary with the same accuracy. In comparison, the L50 mesh is only $8 \mathrm{~m}$ deep at the thalweg compared to almost $14 \mathrm{~m}$ in the REF and C 0.5 meshes. Notice that other less pronounced thalwegs are not captured by $\mathrm{C} 0.5$ due to the application of coarser resolution. 


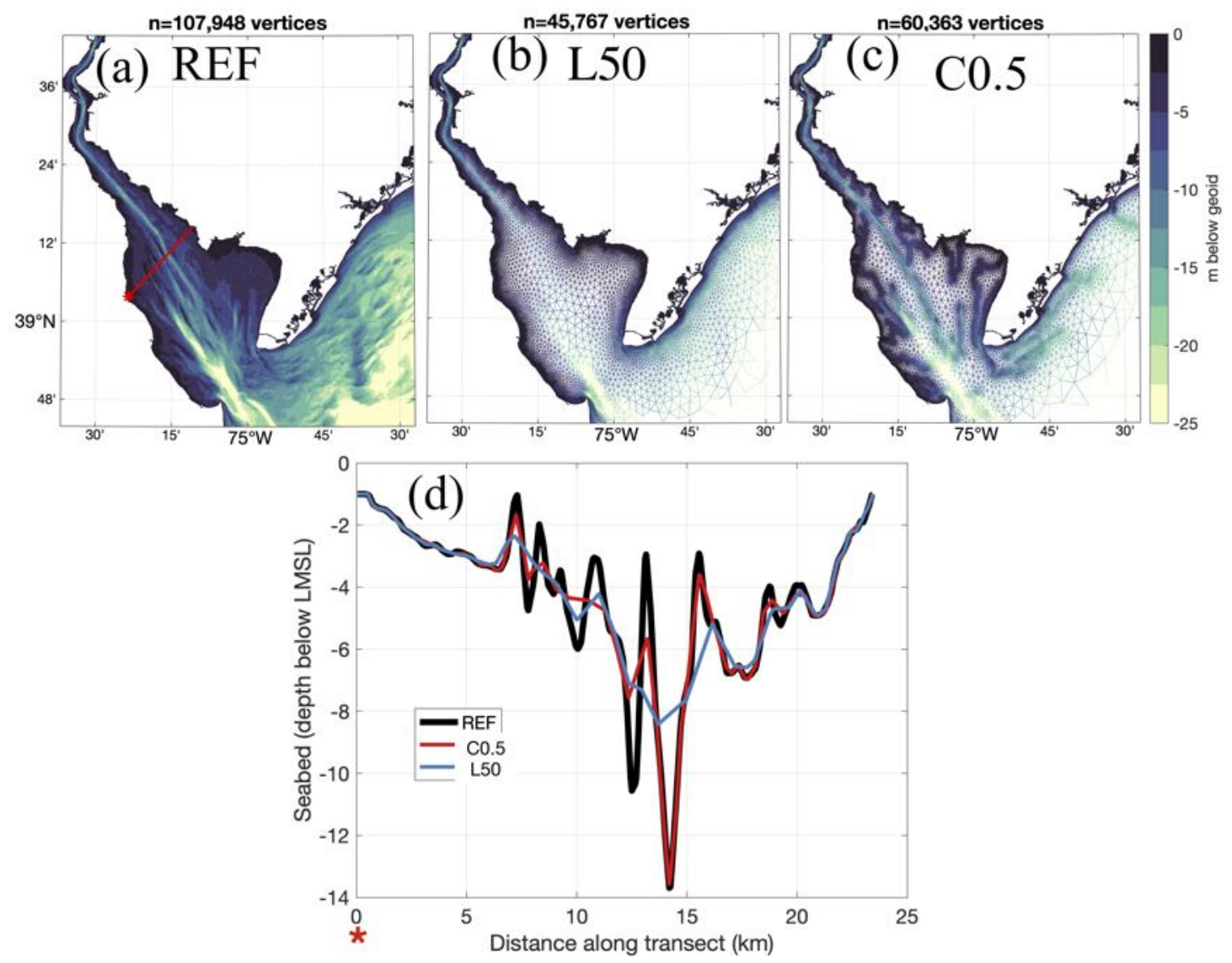

Figure 13. Panels (a)-(c) show sections of meshes in the Delaware Bay estuary and their interpolated seabed data to demonstrate the effect of variably resolving channelized seabeds. Panel (d) illustrates the cross-sectional profile of a tidal channel that is annotated as a red line in panel (a). 

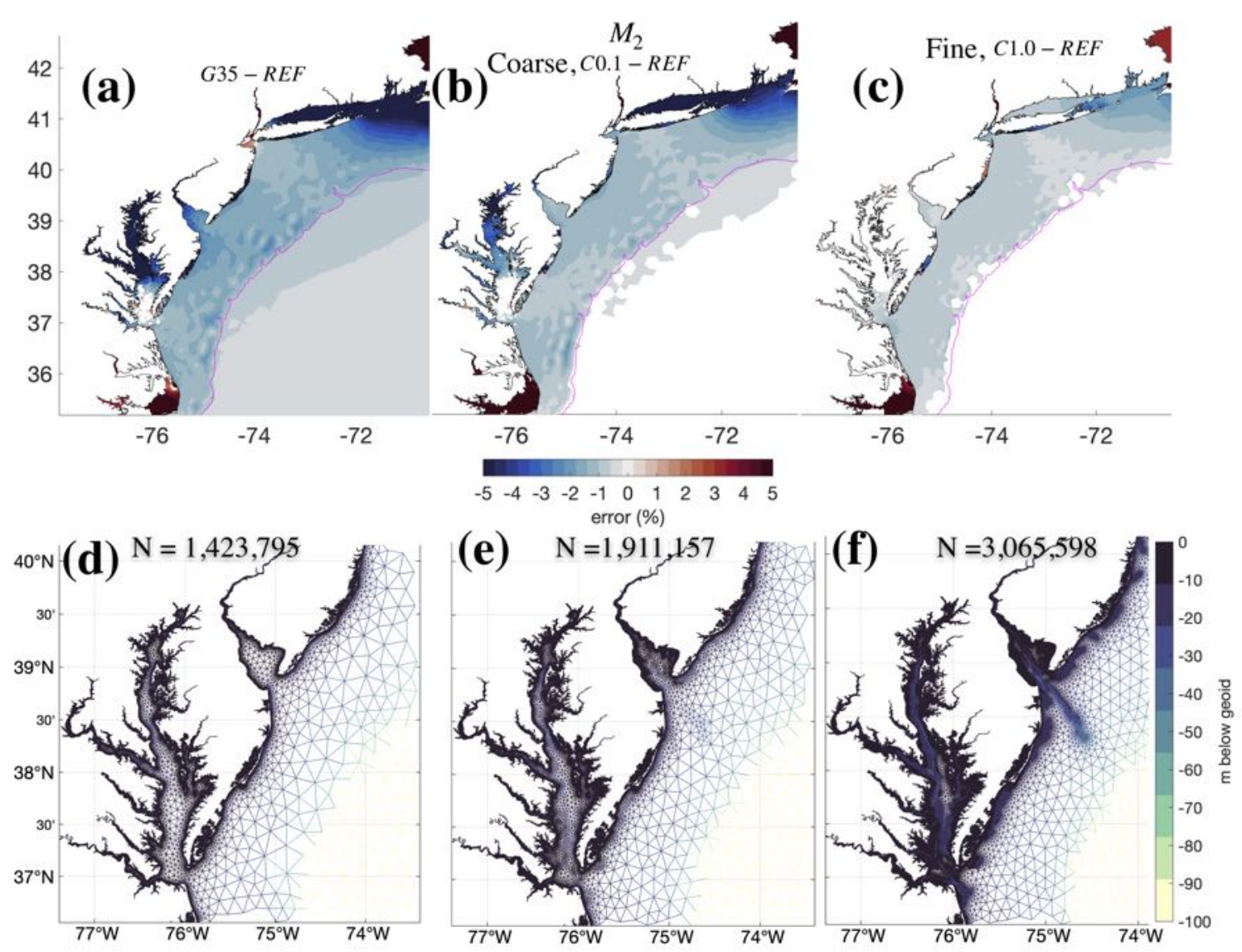

Figure 14. Panels (a)-(c) illustrate the error in the $\mathrm{M}_{2}$ elevation amplitudes for solutions computed on meshes that variably resolve drainage networks that approximate tidal channels. Panels (d)-(f) indicate the mesh and interpolated seabed topography onto the mesh vertices. On panels (d)-(f), the total vertex count is annotated. Note the vertex count indicated in panels (d)-(f) represents the total vertex count in the mesh.

The effects of the estuarine channel mesh size function have been investigated in Experiment $5(\mathrm{C} x)$ using a mesh size gradation of $35 \%(\mathrm{G} 35)$. A higher mesh size gradation motivates the resolution targeting approach because mesh element sizes are relaxed quickly away from the channel thalwegs where finer resolution is applied, thus obtaining a mesh with overall fewer vertices than without the targeting approach. Furhtermore, a lower mesh size gradation (e.g., 15\%) would lead to finer resolution in the center of the estuary where the thalweg may be located and may already adequately resolve the channels cross-sectional profile. The mesh vertex count in the finest $\mathrm{C} x$ mesh (C1.0) is increased by more than two-fold from the G35 mesh to approximately 3.1 million vertices (Figure 14d-f), still approximately $60 \%$ of the G15 mesh vertex count.

The refinement of the estuarine channel network primarily impacts the $\mathrm{M}_{2}$ elevation amplitude solution locally in the estuarine regions of the MAB and NA subdomains (Figure 14a-c). A consistent reduction in $\mathrm{M}_{2} \mathrm{RE}$ from the high mesh size gradation solution (G35) is observed locally, particularly the 5-10\% RE under-prediction error in large estuaries such as the Chesapeake Bay, Delaware Bay, and Long Island Sound. The remaining under-prediction error in these large estuaries is under 1-2\% RE for the C1.0 mesh. Some smaller-scale estuarine systems also exhibit reduction to the RE. For example, the large negative error for G35 (<-5\% RE) in Barnegat Bay (c.f., Figure 1) in the MAB region is reduced to the point that the error changes sign for $\mathrm{C} 1.0(+1$ 2\% RE) (Figure 14a-c). 

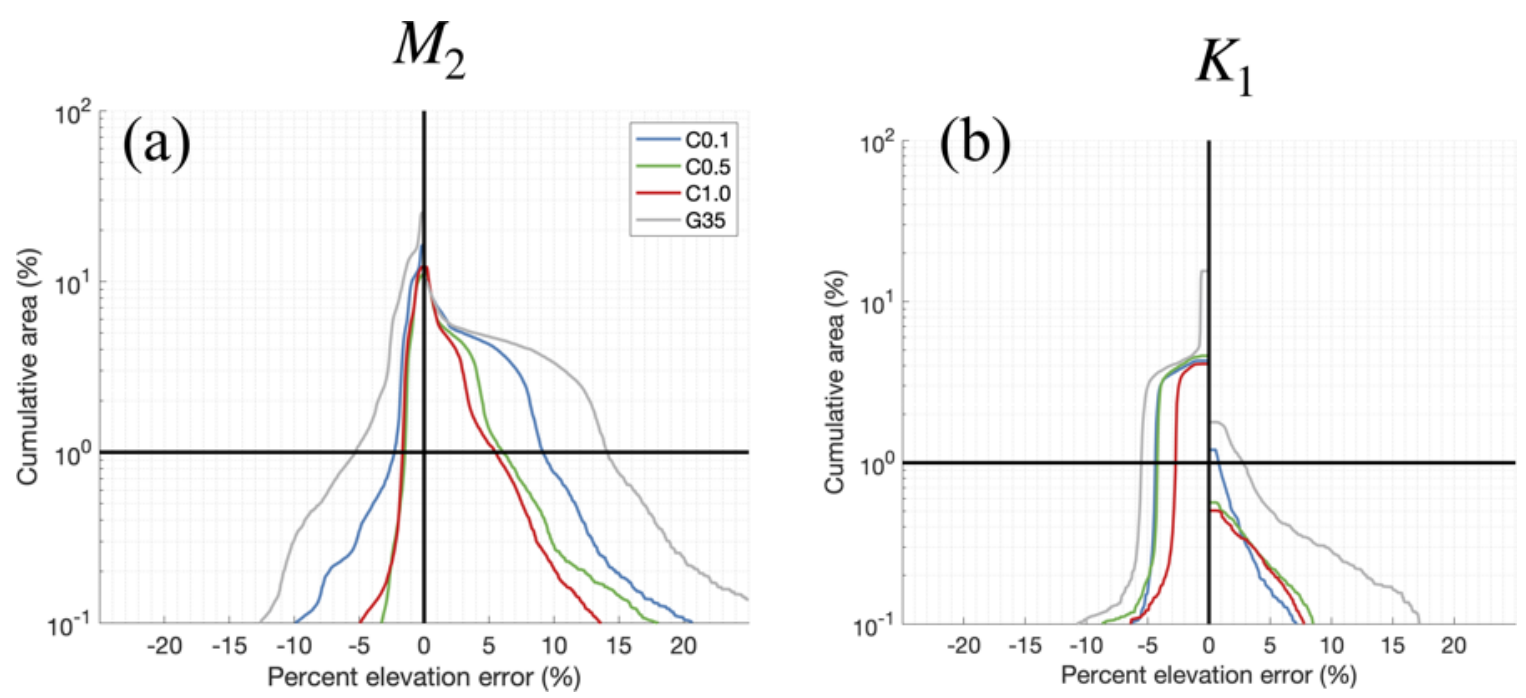

Figure 15. The cumulative area fraction error (CAFE) curves in the comparison zone for the $\mathrm{C} x$ meshes.

Similarly, the CAFE curves also demonstrate a consistent reduction in $\mathrm{M}_{2}$ and $\mathrm{K}_{1} \mathrm{RE}$ in the comparison zone for the $\mathrm{C} x$ meshes and a substantial reduction of $\mathrm{RE}$ as compared to the solution computed on G35 (Figure 15). While none of the meshes have converged with the application of resolution along estuarine channels, the sequence exhibits convergence. Despite the approximately 0.7 million vertex count difference between the C0.5 (2.4 million vertices in total) and C1.0 (3.1 million vertices in total) meshes, their associated solutions perform similarly suggesting diminishing performance gains with finer resolution along estuarine channels. In $99 \%$ of the comparison zone, the $\mathrm{C} 1.0$ mesh $\mathrm{M}_{2}$ error ranges between $-1.6 \%$ and $+5.5 \% \mathrm{RE}$, and $-2.8 \%$ to $+0 \% \mathrm{RE}$ for the $\mathrm{K}_{1}$ producing non-converged solutions for the $\mathrm{M}_{2}$ but converged solutions for the $\mathrm{K}_{1}$. Nevertheless, the narrowing of the error range in $99 \%$ of the comparison zone for the $\mathrm{C} x$ meshes over that of the G35 mesh $\left(-5.0 \%\right.$ to $+15 \%$ for $\left.\mathrm{M}_{2}\right)$ even though the same $35 \%$ gradation is employed is substantial.

\subsection{Summary of experiments}

\subsubsection{Predominant variability}

A summary of the variation in amplitude errors throughout the ECGC region in response to changes in mesh resolution from all 15 meshes over the five experiments (Table 1) is summarized by taking the standard deviation $(\sigma)$ of $\mathrm{RE}$ and the dimensional error, $\mathrm{AE}=A_{I D}-A_{R E F}$ (Figure 16). The greatest changes in the $\mathrm{M}_{2}$ elevation amplitudes are collocated with $\mathrm{M}_{2}$ phase convergence zones and amphidromic points (c.f., Figure 1), and in some large and small estuaries such as the Chesapeake Bay and the Delaware Bay. In the Gulf of Maine, NA which is a resonant basin with a large tidal range (2-10 m), $\sigma_{R E}$ is $1-4 \%$ and $\sigma_{A E}$ is well above $2.5 \mathrm{~cm}$ for $\mathrm{M}_{2}$. The $\mathrm{K}_{1}$ differences in the Gulf of Maine are also larger than most other regions. In the GOM which has a small semidiurnal tidal range, $\sigma_{R E}$ is large in the central region around the convergence zone for $\mathrm{M}_{2}$ but this only corresponds to less than around $2 \mathrm{~mm}$ of dimensional variability ( $\sigma_{A E}$ is very small). In general, the $\mathrm{K}_{1}$ is noticeably less responsive to changes in mesh resolution with $\sigma_{R E}$ barely exceeding $1 \%$. The $\mathrm{K}_{1}$ exhibits the greatest variation in the NA subdomain (Gulf of Maine), in large estuaries, and throughout most of the GOM. The relatively small response in the $\mathrm{K}_{1}$ is to be expected given that it is less energetic and has a longer wavelength than the $\mathrm{M}_{2}$, and it does not typically exhibit resonance on wide shelves [Clarke and Battisti, 1981]. 

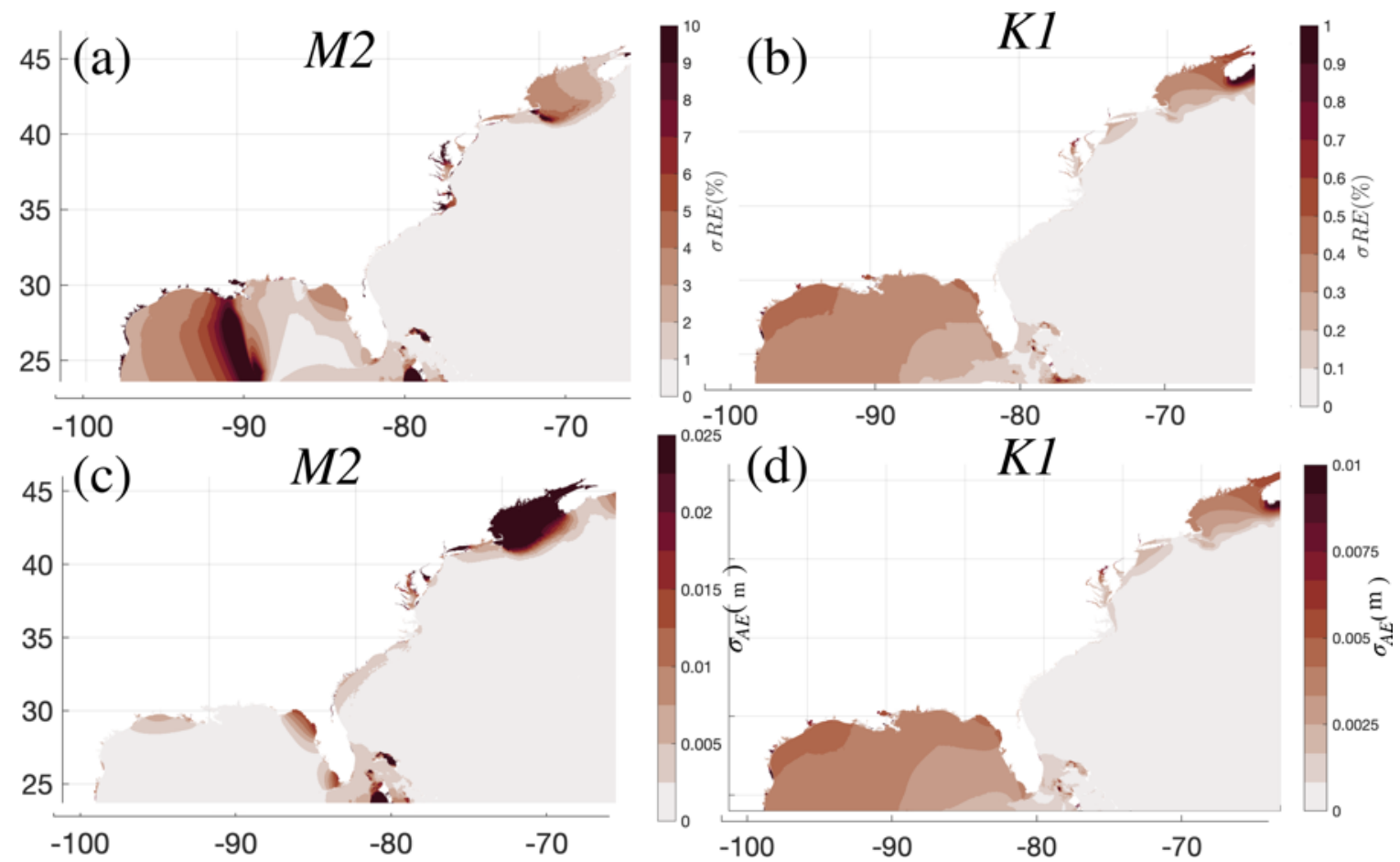

Figure 16. Standard deviation of the relative error $\left(\sigma_{R E}\right)$ in (a) the $\mathrm{M}_{2}$ and (b) the $\mathrm{K}_{1}$ elevation amplitudes for all 15 meshes from the five experiments (Table 1). Panels (c) and (d) are the same but for the standard deviation of the dimensional errors $\left(\sigma_{A E}\right)$. Note the differences smaller than the significance threshold defined in this paper are shown and that the colorbars are not the same between panels (a) and (b). 


\subsubsection{Numerical error versus physical approximation error}

An outstanding issue with the results is that the numerical and physical approximation component of error are intertwined both contributing to the RE observed in the experiments. As the approximation of the bathymetry and shoreline boundary becomes more accurate with the application of finer resolution, the study of convergence in the tidal response becomes challenging as new bathymetric and shoreline features emerge. From a model design point of view, the isolation of the numerical component of the tidal error can provide clarity into how to improve the physical approximation component of error.

To isolate the numerical error in the tidal harmonics studied here, changes in the physical domain approximation was held constant by refining the relatively lightweight L250 mesh so that all triangular edges, except for those within $1^{\circ}$ of the open ocean boundary, were bisected about their midpoints producing four new triangles for every pre-existing one following a shape-preserving scheme [Engwirda, 2014]. The bathymetry from the L250 mesh was linearly interpolated onto this new refined mesh (L250R1) ensuring that the approximation of the seabed topography are identical between the two meshes. Further, the bisection of the elements preserves the representation of the shoreline geometrical features between meshes. The numerical error was then estimated with Richardson extrapolation [Roache, 1994; Blain et al., 1998]. In order to use this approach to estimate numerical truncation error, it was first verified that the leading order error terms indeed controlled the numerical convergence (i.e., asymptotic regime), spatial errors were found to be much greater than the time discretization errors, and the ADCIRC solver in the current configuration was a second order accurate method in space and time [Luettich and Westerink, 2004]. The order of convergence was verified to be 2nd order accurate by refining L250R1 once more producing L250R2.

The Richardson extrapolation base error (REBE) following [Roache, 1994] is calculated to estimate numerical error with the following formulas:

$$
\begin{aligned}
R E B E[\text { coarse mesh }] & =\frac{\epsilon r^{n}}{\left(r^{n}-1\right)} \\
R E B E[\text { fine mesh }] & =\frac{\epsilon}{\left(r^{n}-1\right)} \\
n & =\text { spatial order of ADCIRC }=2 \\
\epsilon & =100 \times \frac{\tilde{f}_{L 250 R 1}-\tilde{f}_{L 250}}{\tilde{f}_{R E F}}[\%] \\
r & =\frac{X_{L 250}}{X_{L 250 R 1}}=2=\text { refinement factor }
\end{aligned}
$$

where $\tilde{f}_{L 250}$ and $\tilde{f}_{L 250 R 1}$ are the solutions computed on the original and refined meshes and $\tilde{f}_{R E F}$ is the solution computed on the reference mesh. $X_{L 250}$ and $X_{L 250 R 1}$ denote the spatially varying mesh sizes throughout the computational domain.

The REBE (herein the numerical error) for the L250 and L250R1 $\mathrm{M}_{2}$ amplitude elevation is presented in Figure 17c,d and compared against the total error that was calculated from the REF solution using Equation 1 (i.e., RE) like was performed in the rest of the paper (Figure 17a,b). There is a similarity in the numerical and total error estimates particularly in the NA subdomain where the magnitude of both errors are 3-5\% for the L250 mesh and diminish to 1-2\% for the L250R1 mesh. However, the estimate of the greatest magnitude numerical error is co-located with the periphery of the Georges Bank near sharp seabed topographic gradients, while the total error is spread across the entire Georges Bank. In general, a weaker reduction in the total error is observed compared to the numerical error. In particular, the total error is not reduced over the Georges Bank or along most of the SAB and MAB coastline (Figure 17a-b). However, the numerical error is reduced almost everywhere to below the significance threshold. For instance, the refinement of L250 to L250R1 reduces the numerical error estimate in the Chesapeake Bay estuary in the MAB re- 

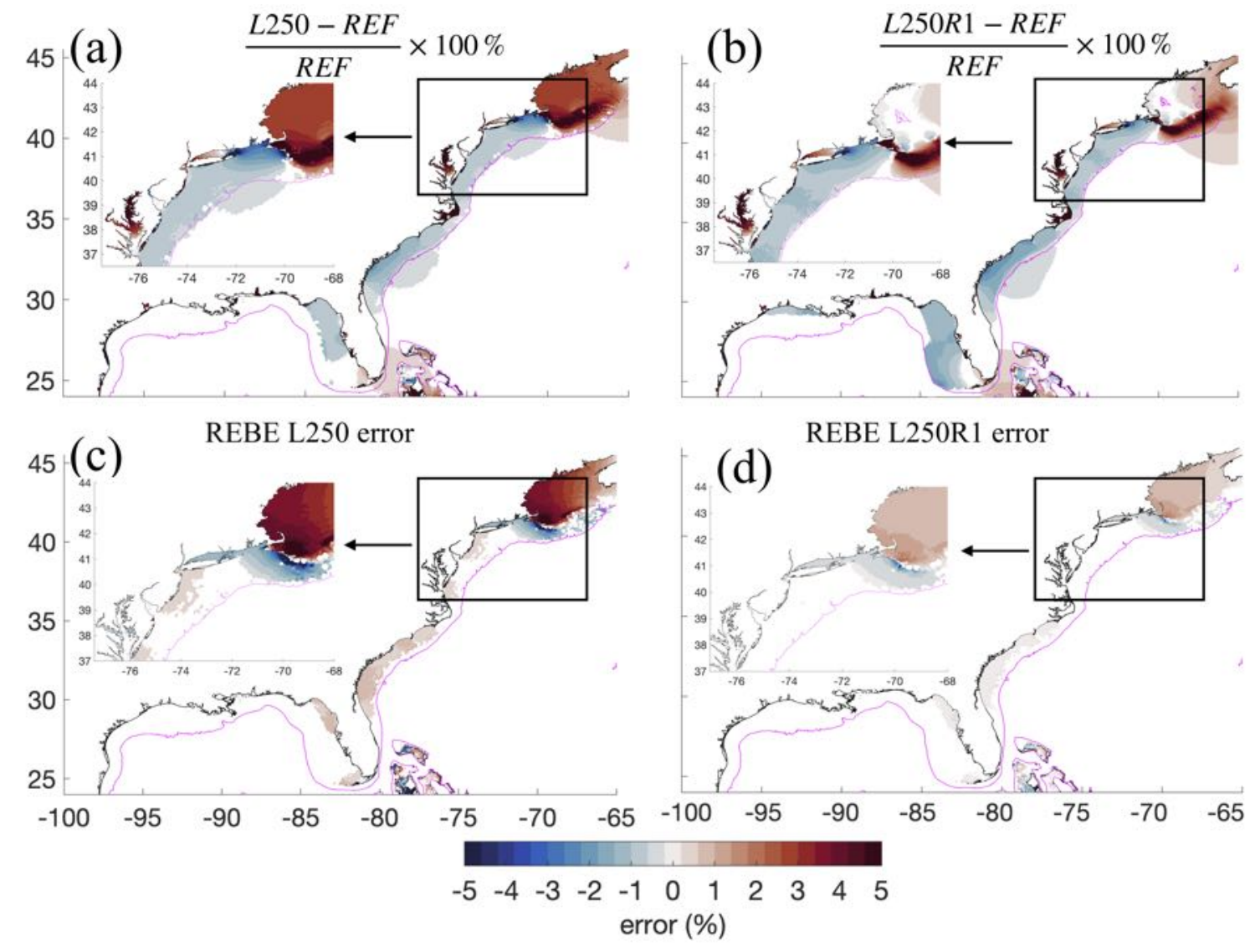

Figure 17. An estimate of the numerical error calculated via Richardson extrapolation following [Roache, 1994] obtained by refining the L250 mesh using a four-to-one refinement strategy to preserve the approximate problem. 
gion markedly. However, the total error does not diminish in the MAB region (particularly the Cheaspeake Bay), which suggests these regions are more responsive to changes in the physical domain approximation (Figure 17). Overall, even though the numerical error has become insignificant (1-2\% in magnitude) and converged as the mesh has been refined, relatively large shoreline and seabed domain approximation errors still remain in the Cheaspeake Bay, the Long Island sound, and the Georges Bank $(\approx 1-5 \%)$. Thus, a method that will reduce the numerical error through an iterative refinement strategy, like LTEA, may still be incapable of improving the accuracy of the solution as compared to observations even if it minimizes the numerical truncation error as it cannot readily incorporate solution responses from un-resolved shoreline geometry scales.

\subsubsection{Mesh design combinations}

The previously described mesh size functions (Table 1) can be used in combination by taking the minimum of each individual function for each point in a regional or global domain [Conroy et al., 2012; Roberts et al., 2018]. Certain combinations of mesh size functions can be regarded as more or less efficient at sufficiently approximating the physical domain. For instance, if the user were to rely on a low mesh size gradation (e.g., 10-15\%), the estuarine channel mesh size function becomes far less necessary because elements in proximity to the channel are already close to the resolution at the shoreline.

Based on our resolution targeting approach, a sequence of mesh designs with different combinations of mesh size functions, all with a high gradation (35\%), were built with the goal of maintaining the accuracy of tidal solution while significantly reducing the vertex count as compared to the REF mesh:

COMBO1: $\min (L 50, S 20)$ with $g \leq 35 \% \rightarrow$ employs 50 -m resolution everywhere along the shoreline (L50), a steep mesh size gradation of $35 \%$ (G35), and enhanced resolution on seabed gradients (S20). A total of 2.3 million vertices.

2: $\min (F S 2, S 20)$ with $g \leq 35 \% \rightarrow$ uses feature size function to vary mesh resolution between $50 \mathrm{~m}$ and $250 \mathrm{~m}$ along the shoreline while maintaining a minimum of two elements across the width of the shoreline (FS2), a steep mesh size gradation of 35\% (G35), and enhanced resolution on seabed gradients (S20). A total of 1.1 million vertices. between $50 \mathrm{~m}$ and $250 \mathrm{~m}$ along the shoreline while maintaining a minimum of two elements across the width of the shoreline (FS2), a steep mesh size gradation of 35\% (G35), enhanced resolution on seabed gradients (S20), and enhanced resolution along estuarine channel features. A total of 1.3 million vertices.

The idea behind this sequence of mesh combinations (COMBOx) is to proceed from a more simple design and move towards a more complex design to test the additive effects, i.e., start with uniform shoreline resolution (COMBO1); use variable shoreline resolution (COMBO2); add additional resolution along estuarine channels (COMBO3). COMBO1 begins with a high gradation rate and a large slope function parameter because of the resolution targeting that we think, and which the experimental results support, lead to more efficient designs. Figure 18 highlighting this targeting approach by illustrating the resolution distribution for the COMBO3 mesh.

Similar to the error reduction patterns in Experiment 4 using fine resolution $(500-1 \mathrm{~km})$ along sharp seabed gradients and a $15 \%$ gradation (c.f., Figure 11), the RE in $\mathrm{M}_{2}$ for all COMBOx meshes is reduced significantly from the G35 mesh, primarily in the NA and MAB subdomains (Figure 19ac). In fact, the S20's CAFE curve is largely similar to the COMBO $x$ meshes. Thus, using S20 to resolve high gradient seabed topographic slopes effectively allows for a much higher elemental size expansion rate to conserve computational resources. Conspicuous positive values of RE near the Georges Bank in proximity to the $\mathrm{M}_{2}$ 's amphidromic point persists, but this is reduced from 10$21 \%$ for the G35 mesh to under 5\% for all COMBOx meshes. The improvement to $\mathrm{M}_{2} \mathrm{RE}$ for the COMBO $x$ meshes is also reflected in their CAFE curves (Figure 19d), which perform similarly to the S20 mesh in $99 \%$ of the comparison zone for the negative crossing (-1\% to $-2 \% \mathrm{RE})$, but con- 


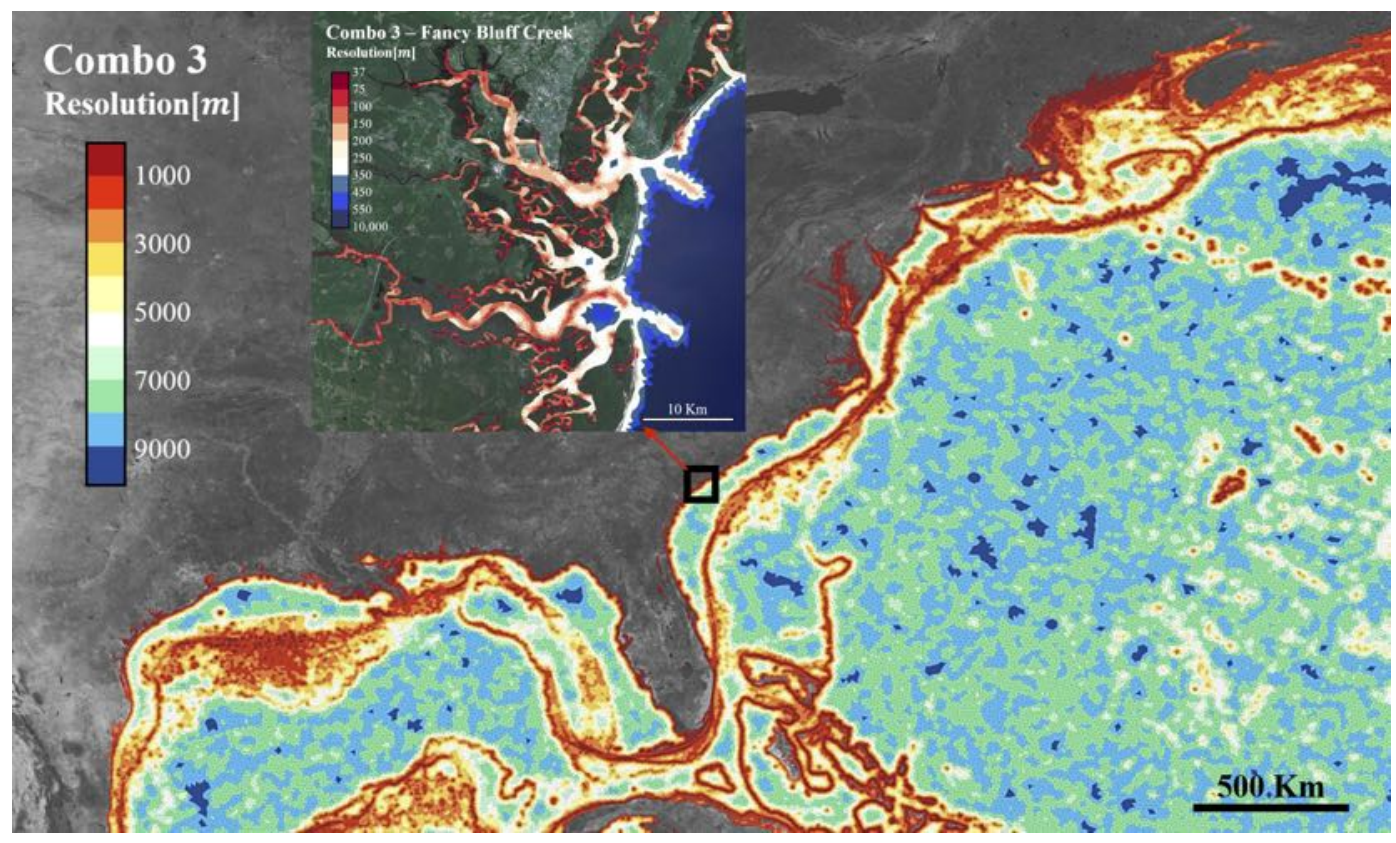

Figure 18. Elemental resolution distribution in the COMBO3 mesh, highlighting how fine resolution is targeted in narrow geometries and along seabed gradients and estaurine channels (see inset in Fancy Bluff Creek).

tain slightly larger RE for the positive crossing ( $+3 \%$ to $+4 \% \mathrm{RE})$. Overall, the RE is substantially reduced from the $+16 \%$ RE positive crossing for the $\mathrm{G} 35$ mesh.

Furthermore, the resulting pattern of errors against measured harmonic data (Figure 20) for the COMBO $x$ meshes approaches that of the REF mesh $\left(B=0.01\right.$ to $0.04, \gamma^{2}=0.03$ to 0.05$)$. In comparison, the positive bias and spread of the errors is significantly greater for the G35 mesh ( $B$ $=0.08, \gamma^{2}=0.33$ ) demonstrating subsatinal improvement in the tidal validation of the mesh with the application of the combinational designs.

The effect on $\mathrm{M}_{2} \mathrm{RE}$ when moving from a uniform shoreline resolution (COMBO1) to variable shoreline resolution $(\mathrm{COMBO} 2)$ based on the feature size approach in the combination meshes is small (Figure 19a-b). Differences less than $1 \%$ are noticeable in the Long Island Sound, Delaware estuary, and around the Georges Bank and Gulf of Maine. Furthermore, the resulting pattern of errors against observations from REF is similar between COMBO1 and COMBO2, although the bias has increased to from 0.01 to 0.04 (Figure 20b-c). Considering that the usage of the FS $x$ shoreline resolution in COMBO2 leads to 53\% fewer vertices than in COMBO1, a small increase to the bias and variance is expected.

The effect on $\mathrm{M}_{2} \mathrm{RE}$ when additional resolution is placed along important estuarine channels (COMBO3 versus COMBO2) can be important in localized regions. The overall picture, as illustrated through the CAFE curves (Figure 19d) and the domain-wide tide gauge error pattern (Figure 20), is relatively unaffected, as evidence by the relatively small change in measured statistics. Predominately, the region of positive RE over the Georges Bank and the Gulf of Maine is increased by approximately $1 \%$ when moving to the COMBO2 and COMBO3 meshes. However, RE is noticeably reduced in the Delaware Bay, Chesapeake Bay, and Long Island Sound to under $+1 \%$ $\mathrm{RE}$ in most areas (Figure 19b-c). Focusing only on the tide gauges $(n=108)$ contained inside the MAB estuaries (Figure. 21), the effect of targeting finer resolution along the channels is further highlighted. The normalized bias is reduced from a positive bias in COMBO2 $(B=0.03)$ to a negative bias for COMBO3 $(B=-0.02)$ inside both estuaries, indicating that COMBO3 performed 


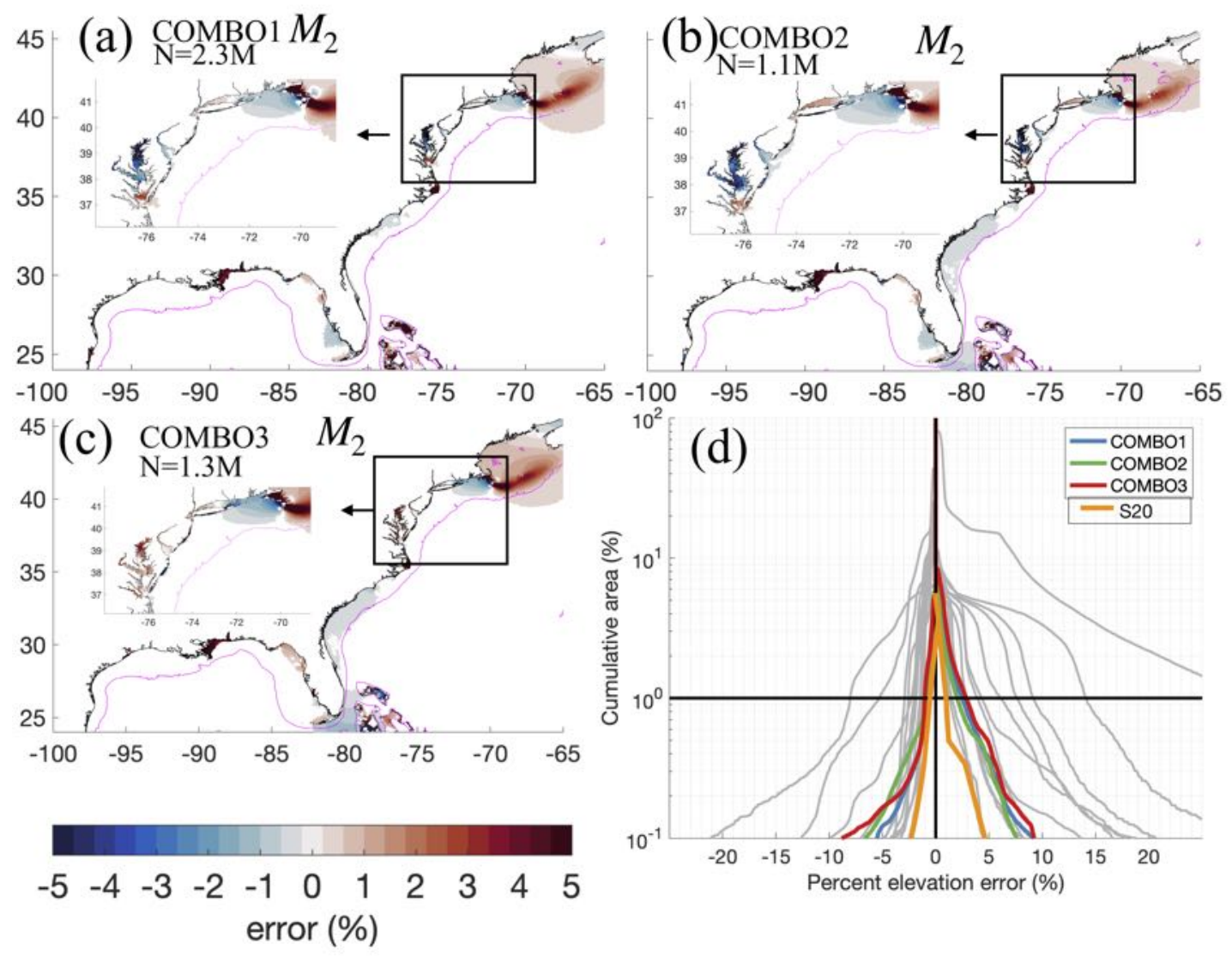

Figure 19. Panels (a)-(c) depict the error in the $\mathrm{M}_{2}$ elevation amplitude solution that was computed on the COMBOx meshes. Panel (d) illustrates a CAFE plot of the error in the comparison zone for the three COMBO $x$ meshes. Grey lines are drawn for other solutions and the S20 mesh (the best performing mesh) is indicated with an orange line. 

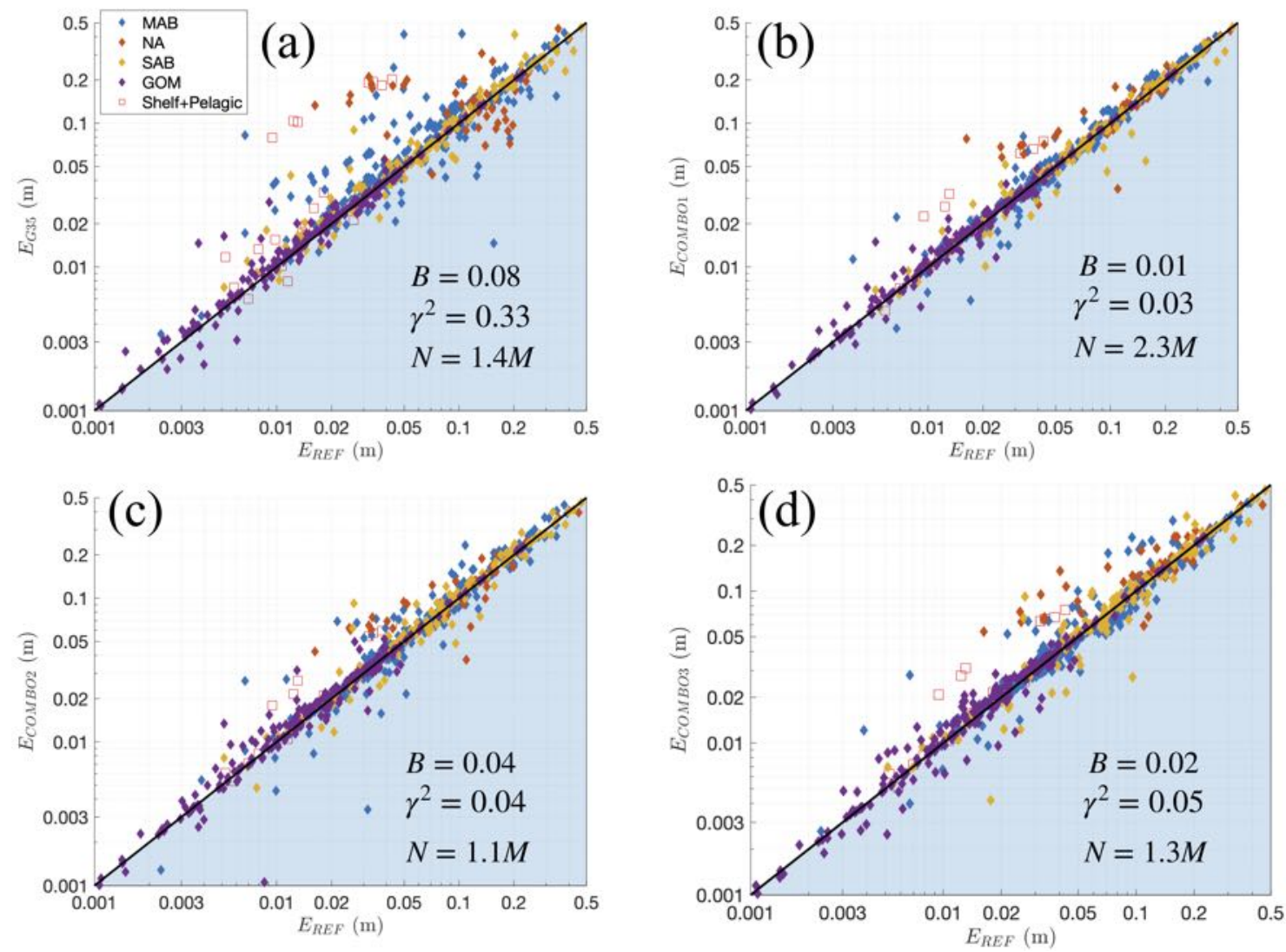

Figure 20. A comparison of the tidal constituent root-mean-square-error $(E)$ for the $\mathrm{M}_{2}$ tidal elevations at 667 tidal gauge observations (c.f., Section 2.1.2) between a solution computed on the REF mesh (x-axis) and the COMBO $x$ meshes (y-axis). The normalized bias $(B)$ and spread $\left(\gamma^{2}\right)$ error metrics and the total vertex $(N)$ are indicated. Points that fall in the blue shaded region have a smaller value of $E$ than the REF solution. Panel (a) is for the G35 solution, panel (b) for COMBO1, panel (c) for COMBO2, and panel (d) for COMBO3. 


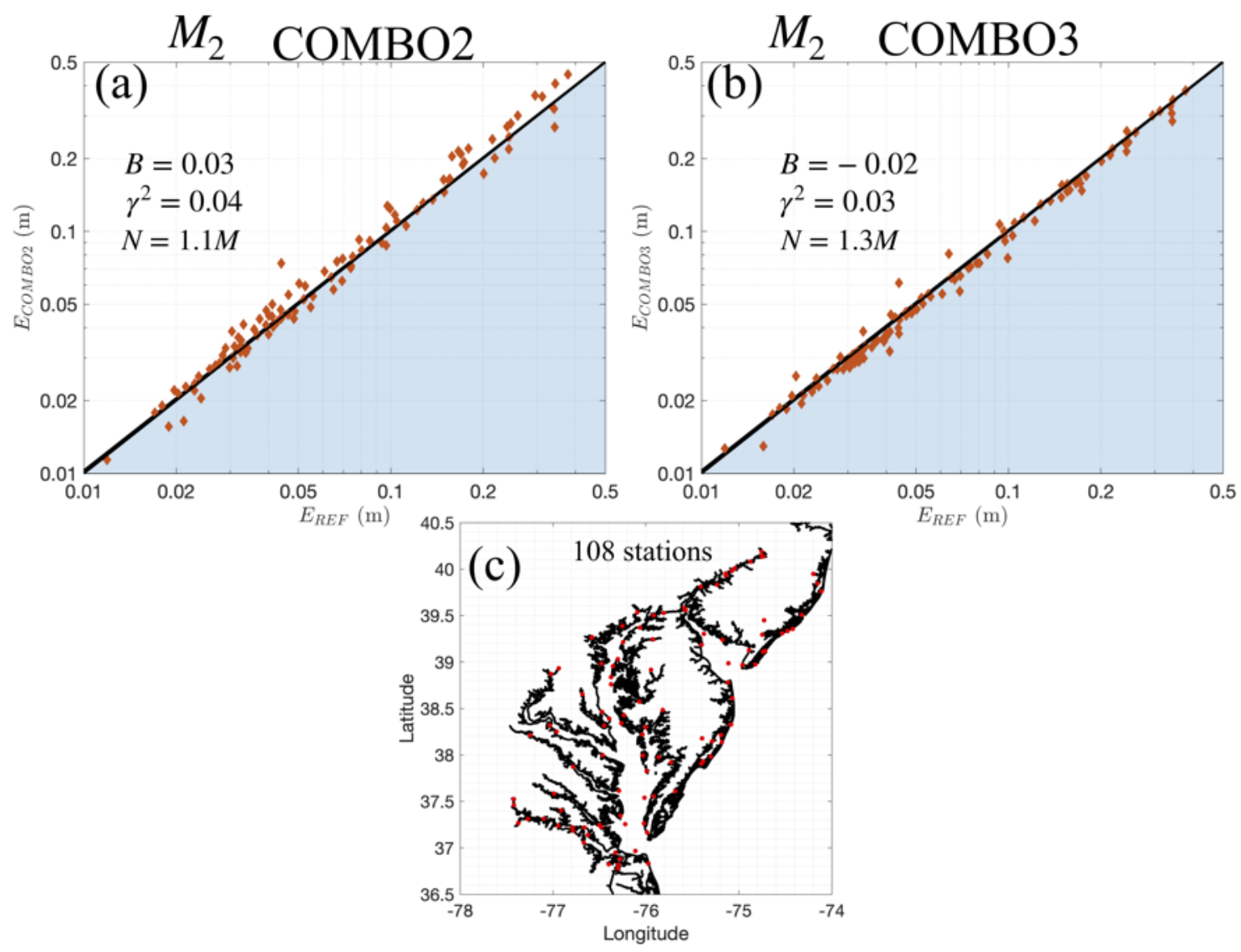

Figure 21. The complex root-mean square error $(E)$ for the solution computed on, (a) the COMBO2 mesh, and (b) the COMBO3 mesh (includes enhanced resolution along estuarine channels), at 108 tide gauges in the Chesapeake Bay and Delaware Bay estuaries that are illustrated in panel (c). Various error metrics are indicated in the panels (a) and (b).

slightly better than the REF mesh here. The normalized spread of the errors $\gamma^{2}$ also reduced but only marginally.

\section{Discussion and Conclusions}

A series of controlled unstructured mesh resolution experiments conducted over a large area of ocean in high-resolution $(\approx 50 \mathrm{~m}$ at the coast) and with a physically accurate shoreline boundary has been achieved through an automatic mesh generation approach facilitated by the OceanMesh2D software [Roberts et al., 2018]. The sensitivity of the barotropic tidal response to unstructured mesh resolution was investigated by controlling the distribution of mesh sizes according to functions of a priori seabed and shoreline geometry information. It is noteworthy to mention that the whole process was scripted and thus automatic using the mesh generator suite. All meshes were designed to be numerically stable with a time step of $2 \mathrm{~s}$ without requiring post-processing handedits (vertex re-location, element re-shaping, or bathymetric smoothing), or ad hoc limiters ${ }^{2}$ and dissipation attributes.

In coastal ocean modeling applications, the shoreline resolution determines the predominate computational expense of the model. We explored ways to quantify the effect of simplifying the

${ }^{2}$ https://wiki.adcirc.org/wiki/Fort.13_file\#Elemental_Slope_Limiter 
shoreline's representation in the mesh by coarsening the minimum resolution from $50 \mathrm{~m}$ to $250 \mathrm{~m}$ and automatically varying the resolution along the shoreline according to the width of shoreline features (Experiment 2, feature size function). Coarsening the minimum resolution ( $\mathrm{L} x$ meshes) noticeably decreased the total area of the mesh by decimating fine scale shoreline features like embayments, headlands, and coves leading to a reduction in the total number of vertices up to a factor of five. However, the associated variation in the tidal elevation amplitudes over most of the domain was comparatively small, the relative errors against the REF solution in $99 \%$ of the domain did not vary by more than $5 \%$, although noticeable differences did occur in the tail of the CAFE plots corresponding to highly localized regions. Experiment 2 demonstrated that the feature size approach FS $x$ preserved the area enclosed by the shoreline of the mesh using the 50-m uniform shoreline resolution (see L50) while requiring approximately half the number of vertices. Further, the relative errors from the REF solution for FS2 showed a significant improvement over L250 in the tail, comparable to L50.

An important point is that the constraints from the sizing functions interact. For example, the increase in feature size parameter from 2 to 8 improves the representation of nearshore seabed topography by using finer resolution across the width of the shoreline feature, but the higher feature size parameter does not improve the ability to resolve the complexity of the shoreline as the minimum element size bound is reached (c.f. Figure 4). Thus, our recommendation is that meshes intended for high-resolution tidal modeling to be constructed with a feature size approach (also see Conroy et al. [2012]) with maximally two or three vertices across the shoreline's width instead of applying a minimum resolution uniformly along the shoreline [Bunya et al., 2010; Kerr et al., 2013]. Note that in the feature size approach, a consideration should be taken to make sure that the element sizes along the shoreline cannot become too coarse. In this work, we applied a five-to-one ratio upper bound so that the element sizes do not exceed $250 \mathrm{~m}$ given that the length scales of the physical processes are still controlled by the proximity to fine scale shoreline geometry here, and coarse element sizes nearshore may not be conducive to accurately model other coastal processes that were not considered in this study such as wave setup induced through wave breaking [Joyce et al., 2019].

Experiment 3 demonstrated how increasing the gradation rate can negatively impact the approximation of seabed topography in the mesh and the simulated accuracy of tidal solutions were highly degraded. The mesh with the highest gradation (G35) was the worst performing mesh in terms of the $\mathrm{M}_{2}$ and $\mathrm{K}_{1}$ relative error values out of all 15 meshes in the five experiments. The effect of increasing the gradation is likely to have increased the numerical error [Hagen et al., 2000] in addition to the physical domain approximation error (e.g., representation of seabed topography) making the determination of the root cause of the poor performance challenging. However, experiment 4 clearly demonstrated that placing resolution along seabed gradients $(\sim 1 \mathrm{~km}$ along the continental shelf break and slope) improved the accuracy of tidal solutions, which is in agreement with prior works [Luettich and Westerink, 1995; Chen et al., 2016]. At the same time, increasing the gradation rate coarsened the representation of the continental shelf break as resolution sizes would grow faster from the shoreline. Thus, it is likely that our application of resolution along seabed gradients reduces the numerical error as large gradients in the solution are co-located with steep seabed topographic gradients [e.g., Hannah and Wright, 1995; Hagen et al., 2001]. Our recommendation is the use of a high value for the slope mesh size function (e.g., S10-S20) in combination with a high gradation rate (e.g., G35) to offset the negative impacts on both error sources, while largely reducing the total number of vertices in the mesh.

Experiment 5 demonstrated that the approximation of the seabed topography across estuaries with deep-draft channels (e.g., Chesapeake Bay and Delaware Bay) could be improved by using the estuarine channel mesh size function to place targeted high-resolution zones along the submarine channels inside and leading into estuaries. In estuaries that are characterized by well-defined submarine channels that occupy non-trivial portions of the width of the estuary, it is important to ensure that adequate resolution is placed along these channels so that the total cross-sectional area and local ocean depth minima are preserved. Indeed, progressively placing finer mesh resolution along the estuarine channel network (extracted using an upslope area computation on the DEM) 
was shown to reduce tidal error metrics as compared to both the reference solution and measured data as inland waterway conveyances are improved and frictional resistance is reduced. We remark that other mesh size heuristics, such as the slope mesh size function and using finer resolution along the shoreline with a low gradation rate can implicitly, but inefficiently, capture these submarine channel features. Thus, the application of the estuarine channel mesh size function allows the usage of a higher mesh size gradation so as to focus resolution only on the submarine channels allowing us to more efficiently discretize the estuarine environment.

We tested the performance of mesh design strategies that involved using a steep mesh size gradation rate (G35) in combination with the targeted mesh sizing functions along the shoreline (FSx), sharp topographic gradients ( $\mathrm{S} x$ ), and estuarine channel systems $(\mathrm{C} x)$. Three combination meshes (COMBOx) that ranged from 1.1 million to 2.3 million vertices were generated. Overall, all COMBO $x$ meshes performed similarly to the REF mesh both directly and as compared to measured tide gauge data. The additive effects of multiple mesh size functions reduced the error metrics largely, especially in the comparison to the G35 solution, which had a noticeably degraded solution without the usage of other sizing functions (in particular the slope function) used in the COMBO $x$ sequence.

Echoing our findings from Experiment 1, the COMBO2 mesh utilized a small value of the feature size function parameter (FS2) and had approximately half the vertex count of COMBO1 (uniform shoreline resolution) with little increase in relative error, thus the FS2 is considered an efficient mesh design choice. However, deep-draft channels within estuarine are more likely to poorly represented with the high gradation (G35) and FSx design combination as mesh sizes will become coarser in certain regions depending on the cuspate shape of the shoreline. Our conclusion is the $15 \%$ increase in the total vertex count associated with the addition of the $\mathrm{C} 0.5 \mathrm{com}$ ponent of COMBO3 to better capture estuarine channels, can be considered a good investment particularly since the solution in nearshore estuaries of high importance is improved; even to a point beyond the performance of the REF mesh (e.g., Figure 21). Our results imply that the 250-m bounded blanket resolution applied across the large estuaries in reference solution is coarser and less effective than the targeted resolution that follows the channelized seabed in the $\mathrm{C} 0.5$ mesh size function. In fact, a key drawback of mesh designs that apply uniformly fine zones of resolution throughout regions of similar ocean depths (the wavelength-to-gridscale heuristic [e.g., Westerink et al., 1994] is that there is less flexibility to more finely capture targeted seabed features and shoreline constrictions due to the baseline expense of the model. In many regions, the application of targeted refinement can produce more finely resolved solutions in localized areas of importance with far fewer vertices.

Through the combination of the constraints imposed by a set of mesh size functions (COMBOx meshes), the vertex count was reduced by nearly an order of magntiude from the reference mesh and had a converged solution with tidal error metrics in $99 \%$ of the East and Gulf Coast waters ranging from $-2 \%$ to $+1 \%$. For instance $\mathrm{COMBO} 3$ (1.3 million vertices) had eight times fewer vertices as the reference (10.8 million vertices). These results suggest that pre-existing operational models may be largely inefficient, over-discretizing in some areas and under-discretizing in others as pre-existing models use nearly uniform resolution nearshore and land and following the wavelength-to-gridscale sizing heuristic offshore. For example, the Hurricane Surge Operational Forecasting system (HSOFS) mesh [Technology Riverside Inc. and AECOM, 2015] used in realtime predictions employs a minimum shoreline resolution of $250 \mathrm{~m}$ and contains 0.75 million underwater vertices, which is similar in number to our L250 mesh. In contrast, the COMBO3 mesh, which spans the same ECGC study region, utilizes up to five times finer resolution nearshore (50 $\mathrm{m}$ compared to $250 \mathrm{~m}$ ) and up to ten times finer resolution along the continental slope $(1 \mathrm{~km} \mathrm{com}-$ pared to $10 \mathrm{~km}$ ), with only 1.6 times the total number of underwater vertices than HSOFS.

We highlight that an important first step in the coastal model development procedure is to construct a mesh that minimizes the physical domain approximation error before model tuning occurs vis-a-vis varying bottom friction, other dissipative coefficients, viscous models, and manually altering ocean depths and shoreline form. As was evident in this paper, by improving the accuracy of the approximate problem (i.e., the representation of the shoreline and seabed topography 
as per the available geospatial data used), the tidal solutions exhibited convergence towards a reference solution. The primary variation in the $\mathrm{M}_{2}$ (c.f., Figure 16) tended to coincide with zones of the ECGC in which the bottom friction coefficient are typically modified [Szpilka et al., 2016]. For instance, since the Chesapeake Bay has a muddy seabed floor, the friction coefficient, $C_{f}$ is often set low a value $\left(C_{f} \approx 0.001\right)$ and this is found to improve comparisons with tidal harmonics [Friedrichs, 2010]. However, our results indicate that the the $\mathrm{M}_{2}$ tide in the Chesapeake estuary is largely sensitive to mesh design with changes around $15 \%$ between the mesh design variations explored here (c.f., Figure 16). It is thus likely that the bottom friction application procedure may be tuned incorrectly depending on the local mesh design; for instance, depending on the complexity of the estuarine network in the mesh.

This study highlights the need to perform convergence stuides to determine the role of mesh resolution on solutions of coastal hydrodynamics. We have provided a framework to perform these convergence studies and also suggestions for starting mesh size function parameters (e.g., COMBO3) and the effect they have on the solution of surface tides.

\section{Acknowledgments}

This work was supported by the Joseph and Nona Ahearn endowment at the University of Notre Dame and was completed under USDOC NOAA Award NA18OAR4590377. OceanMesh2D V2.0 is available from https://doi.org/10.5281/zenodo.2560555.

\section{References}

Amante, C., and B. Eakins (2009), ETOPO1 1 Arc-Minute Global Relief Model: Procedures, Data Sources and Analysis. NOAA Technical Memorandum NESDIS NGDC-24. National Geophysical Data Center, NOAA., doi:10.7289/V5C8276M.

Avdis, A., A. S. Candy, J. Hill, S. C. Kramer, and M. D. Piggott (2018), Efficient unstructured mesh generation for marine renewable energy applications, Renewable Energy, 116, 842 - 856, doi:10.1016/j.renene.2017.09.058.

Bacopoulos, P., and S. C. Hagen (2017), The intertidal zones of the south atlantic bight and their local and regional influence on astronomical tides, Ocean Modelling, 119, 13 - 34, doi:10.1016/j. ocemod.2017.09.002.

Beardsley, R. C., C. Chen, and Q. Xu (2013), Coastal flooding in Scituate (MA): A FVCOM study of the 27 December 2010 nor'easter, Journal of Geophysical Research: Oceans, 118(11), 60306045, doi:10.1002/2013JC008862.

Behrens, J. (1998), Atmospheric and ocean modeling with an adaptive finite element solver for the shallow-water equations, Applied Numerical Mathematics, 26(1), 217 - 226, doi:10.1016/S01689274(97)00090-1.

Bilgili, A., K. W. Smith, and D. R. Lynch (2006), BatTri: A two-dimensional bathymetry-based unstructured triangular grid generator for finite element circulation modeling, Computers and Geosciences, 32(5), 632-642, doi:10.1016/j.cageo.2005.09.007.

Blain, C. A., J. J. Westerink, and R. A. Luettich (1998), Grid convergence studies for the prediction of hurricane storm surge, International Journal for Numerical Methods in Fluids, 26(4), 369401, doi:10.1002/(SICI)1097-0363(19980228)26:4<369::AID-FLD624>3.0.CO;2-0.

Blanton, B. O., F. E. Werner, H. E. Seim, R. A. Luettich, D. R. Lynch, K. W. Smith, G. Voulgaris, F. M. Bingham, and F. Way (2004), Barotropic tides in the South Atlantic Bight, Journal of Geophysical Research C: Oceans, 109(12), 1-17, doi:10.1029/2004JC002455.

Bunya, S., J. C. Dietrich, J. J. Westerink, B. A. Ebersole, J. M. Smith, J. H. Atkinson, R. Jensen, D. T. Resio, R. A. Luettich, C. Dawson, V. J. Cardone, A. T. Cox, M. D. Powell, H. J. Westerink, and H. J. Roberts (2010), A High-Resolution Coupled Riverine Flow, Tide, Wind, Wind Wave, and Storm Surge Model for Southern Louisiana and Mississippi. Part I: Model Development and Validation, Monthly Weather Review, 138(2), 345-377, doi:10.1175/2009MWR2906.1.

Candy, A., and J. Pietrzak (2018), Shingle 2.0: Generalising self-consistent and automated domain discretisation for multi-scale geophysical models, Geoscientific Model Development, pp. 213- 
234, doi:10.5194/gmd-11-213-2018.

Chen, C., H. Huang, R. C. Beardsley, Q. Xu, R. Limeburner, G. W. Cowles, Y. Sun, J. Qi, and H. Lin (2011), Tidal dynamics in the Gulf of Maine and New England Shelf: An application of FVCOM, Journal of Geophysical Research: Oceans, 116(12), 1-14, doi:10.1029/2011JC007054.

Chen, C., R. C. Beardsley, R. A. Luettich, J. J. Westerink, H. Wang, W. Perrie, Q. Xu, A. S. Donahue, J. Qi, H. Lin, L. Zhao, P. C. Kerr, Y. Meng, and B. Toulany (2013), Extratropical storm inundation testbed: Intermodel comparisons in Scituate, Massachusetts, Journal of Geophysical Research: Oceans, 118(10), 5054-5073, doi:10.1002/jgrc.20397.

Chen, C., G. Gao, Y. Zhang, R. C. Beardsley, Z. Lai, J. Qi, and H. Lin (2016), Circulation in the Arctic Ocean: Results from a high-resolution coupled ice-sea nested Global-FVCOM and ArcticFVCOM system, Progress in Oceanography, 141, 60-80, doi:10.1016/j.pocean.2015.12.002.

Clarke, A. J., and D. S. Battisti (1981), The effect of continental shelves on tides, Deep Sea Research Part A. Oceanographic Research Papers, 28(7), 665 - 682, doi:10.1016/0198-0149(81) 90128-X.

Conroy, C. J., E. J. Kubatko, and D. W. West (2012), ADMESH:An advanced, automatic unstructured mesh generator for shallow water models, Ocean Dynamics, 62(10-12), 1503-1517, doi: 10.1007/s10236-012-0574-0.

Cyriac, R., J. Dietrich, J. Fleming, B. Blanton, C. Kaiser, C. Dawson, and R. Luettich (2018), Variability in Coastal Flooding predictions due to forecast errors during Hurricane Arthur, Coastal Engineering, 137, 59 - 78, doi:10.1016/j.coastaleng.2018.02.008.

Dietrich, J. C., S. Bunya, J. J. Westerink, B. A. Ebersole, J. M. Smith, J. H. Atkinson, R. Jensen, D. T. Resio, R. A. Luettich, C. Dawson, V. J. Cardone, A. T. Cox, M. D. Powell, H. J. Westerink, and H. J. Roberts (2010), A High-Resolution Coupled Riverine Flow, Tide, Wind, Wind Wave, and Storm Surge Model for Southern Louisiana and Mississippi. Part II: Synoptic Description and Analysis of Hurricanes Katrina and Rita, Monthly Weather Review, 138(2), 378404, doi:10.1175/2009MWR2907.1.

Dietrich, J. C., J. J. Westerink, A. B. Kennedy, J. M. Smith, R. E. Jensen, M. Zijlema, L. H. Holthuijsen, C. Dawson, R. A. Luettich, M. D. Powell, V. J. Cardone, A. T. Cox, G. W. Stone, H. Pourtaheri, M. E. Hope, S. Tanaka, L. G. Westerink, H. J. Westerink, and Z. Cobell (2011), Hurricane gustav (2008) waves and storm surge: Hindcast, synoptic analysis, and validation in southern louisiana, Monthly Weather Review, 139(8), 2488-2522, doi:10.1175/2011MWR3611.1.

Dronkers, J. (1986), Tidal asymmetry and estuarine morphology, Netherlands Journal of Sea Research, 20(2), 117 - 131, doi:10.1016/0077-7579(86)90036-0.

Engwirda, D. (2014), Locally optimal Delaunay-refinement and optimisation-based mesh generation, Ph.D. thesis, University of Sydney.

Engwirda, D. (2017), JIGSAW-GEO (1.0): Locally orthogonal staggered unstructured grid generation for general circulation modelling on the sphere, Geoscientific Model Development, 10(6), 2117-2140, doi:10.5194/gmd-10-2117-2017.

Fleming, J. G., C. W. Fulcher, R. a. Luettich, B. D. Estrade, G. D. Allen, and H. S. Winer (2008), A Real Time Storm Surge Forecasting System Using ADCIRC, in 10th International Conference on Estuarine and Coastal Modeling, pp. 893-912, Newport, Rhode Island, doi:10.1061/ $40990(324) 48$.

Friedrichs, C. T. (2010), Barotropic tides in channelized estuaries, in Contemporary Issues in Estuarine Physics, edited by A. Valle-Levinson, pp. 27-61, Cambridge University Press, doi: 10.1017/CBO9780511676567.004.

Garrett, C., and E. Kunze (2007), Internal Tide Generation in the Deep Ocean, Annual Review of Fluid Mechanics, 39(1), 57-87, doi:10.1146/annurev.fluid.39.050905.110227.

Gorman, G., M. Piggott, M. Wells, C. Pain, and P. Allison (2008), A systematic approach to unstructured mesh generation for ocean modelling using gmt and terreno, Computers and Geosciences, 34(12), 1721-1731, doi:10.1016/j.cageo.2007.06.014.

GRASS Development Team (2017), Geographic Resources Analysis Support System (GRASS GIS) Software, Version 7.2, Open Source Geospatial Foundation.

Green, J. A. M., and J. Nycander (2013), A Comparison of Tidal Conversion Parameterizations for Tidal Models, Journal of Physical Oceanography, 43(1), 104-119, doi:10.1175/JPO-D-12-023.1. 
Greenberg, D. A., F. Dupont, F. H. Lyard, D. R. Lynch, and F. E. Werner (2007), Resolution issues in numerical models of oceanic and coastal circulation, Continental Shelf Research, 27(9), 13171343, doi:10.1016/j.csr.2007.01.023.

Hagen, S. C., J. J. Westerink, and R. L. Kolar (2000), One-dimensional finite element grids based on a localized truncation error analysis, International Journal for numerical methods in fluids, 32, 241-261, doi:10.1002/(SICI)1097-0363(20000130)32:2<241::AID-FLD947>3.0.CO;2- $\{\backslash \#$.

Hagen, S. C., J. J. Westerink, R. L. Kolar, and O. Horstmann (2001), Two-dimensional, unstructured mesh generation for tidal models, International Journal for Numerical Methods in Fluids, 35(6), 669-686, doi:10.1002/1097-0363(20010330)35:6<669::AID-FLD108>3.0.CO;2- \{\\# .

Hagen, S. C., O. Horstmann, and R. J. Bennett (2002), An unstructured mesh generation algorithm for shallow water modeling, International Journal of Computational Fluid Dynamics, 16(2), 8391, doi:10.1080/10618560290017176.

Hannah, C., and D. Wright (1995), Depth dependent analytical and numerical solutions for winddriven flow in the coastal ocean, Quantitative Skill Assessment for Coastal Ocean Models, 47, $125-152$.

Hendershott, M. C. (1972), The Effects of Solid Earth Deformation on Global Ocean Tides, Geophysical Journal International, 29(4), 389-402, doi:10.1111/j.1365-246X.1972.tb06167.x.

Hope, M. E., J. J. Westerink, A. B. Kennedy, P. C. Kerr, J. C. Dietrich, C. Dawson, C. J. Bender, J. M. Smith, R. E. Jensen, M. Zijlema, L. H. Holthuijsen, R. A. Luettich, M. D. Powell, V. J. Cardone, A. T. Cox, H. Pourtaheri, H. J. Roberts, J. H. Atkinson, S. Tanaka, H. J. Westerink, and L. G. Westerink (2013), Hindcast and validation of Hurricane Ike (2008) waves, forerunner, and storm surge, Journal of Geophysical Research: Oceans, 118(9), 4424-4460, doi:10.1002/jgrc. 20314.

Huthnance, J. M. (1995), Circulation, exchange and water masses at the ocean margin: the role of physical processes at the shelf edge, Progress in Oceanography, 35(4), 353 - 431, doi:10.1016/ 0079-6611(95)80003-C.

Joyce, B., J. Gonzalez-Lopez, A. J. van der Westhuysen, D. Yang, W. J. Pringle, J. J. Westerink, and A. T. Cox (2019), U.S. IOOS Coastal and Ocean Modeling Testbed: Hurricane-induced Winds, Waves and Surge for Deep-ocean, Reef Fringed Islands in the Caribbean, Journal of Geophysical Research C: Oceans, doi:10.1029/2018JC014687.

Kerr, P. C., R. C. Martyr, A. S. Donahue, M. E. Hope, J. J. Westerink, R. A. Luettich, A. B. Kennedy, J. C. Dietrich, C. Dawson, and H. J. Westerink (2013), U.S. IOOS coastal and ocean modeling testbed: Evaluation of tide, wave, and hurricane surge response sensitivities to mesh resolution and friction in the Gulf of Mexico, Journal of Geophysical Research: Oceans, 118(9), 4633-4661, doi:10.1002/jgrc.20305.

Kinnmark, I. (1988), The shallow water wave equations: Formulation, analysis and application, SIAM Review, 30(3), 517-518, doi:10.1137/1030116.

Loder, J. W. (1980), Topographic Rectification of Tidal Currents on the Sides of Georges Bank, Journal of Physical Oceanography, 10(9), 1399-1416, doi:10.1175/1520-0485(1980)010<1399: TROTCO $>2.0 . \mathrm{CO} ; 2$.

Luettich, R., and J. J. Westerink (2004), Formulation and Numerical Implementation of the 2D/3D ADCIRC Finite Element Model Version 44.XX, Tech. rep.

Luettich, R., Jr, and J. J. Westerink (1995), Continental Shelf Scale Convergence Studies with a Barotropic Tidal Model, Quantitative Skill Assessment for Coastal Ocean Models, A.G.U., 47, doi: 10.1029/CE047p0349.

Luettich, R. A., and J. J. Westerink (2013), Continental Shelf Scale Convergence Studies with a Barotropic Tidal Model, chap. 16, pp. 349-371, American Geophysical Union (AGU), doi: 10.1029/CE047p0349.

Lyard, F., F. Lefevre, T. Letellier, and O. Francis (2006), Modelling the global ocean tides: modern insights from FES2004, Ocean Dynamics, 56(5-6), 394-415, doi:10.1007/s10236-006-0086-x.

Lynch, D. R., and W. G. Gray (1979), A wave equation model for finite element tidal computations, Computers Fluids, 7(3), 207 - 228, doi:10.1016/0045-7930(79)90037-9.

Marsooli, R., and N. Lin (2018), Numerical Modeling of Historical Storm Tides and Waves and Their Interactions Along the U.S. East and Gulf Coasts, Journal of Geophysical Research: 
Oceans, pp. 3844-3874, doi:10.1029/2017JC013434.

Massey, T. C. (2015), Locally constrained nodal connectivity refinement procedures for unstructured triangular finite element meshes, Engineering with Computers, 31(2), 375-386, doi: 10.1007/s00366-014-0357-y.

Molines, J., M. Fornerino, and C. L. Provost (1989), Tidal spectroscopy of a coastal area: observed and simulated tides of the lake maracaibo system, Continental Shelf Research, 9(4), $301-323$, doi:10.1016/0278-4343(89)90036-8.

Muis, S., N. Lin, M. Verlaan, H. C. Winsemius, P. J. Ward, and J. C. J. H. Aerts (2019), Spatiotemporal patterns of extreme sea levels along the western North-Atlantic coasts, Scientific Reports, 9(1), 3391, doi:10.1038/s41598-019-40157-w.

Oden, J., L. Demkowicz, W. Rachowicz, and T. Westermann (1990), A posteriori error analysis in finite elements: The element residual method for symmetrizable problems with applications to compressible euler and navier-stokes equations, Computer Methods in Applied Mechanics and Engineering, 82(1), 183 - 203, doi:10.1016/0045-7825(90)90164-H, proceedings of the Workshop on Reliability in Computational Mechanics.

Parker, B. B. (1991), The relative importance of the various nonlinear mechanisms in a wide range of tidal interactions (Review), in Tidal hydrodynamics, edited by B. B. Parker, pp. 237-268, John Wiley \& Sons, Inc., New York, USA.

Parrish, D. M., and S. C. Hagen (2009), Incorporating spatially variable bottom stress and Coriolis force into 2D, a posteriori , unstructured mesh generation for shallow water models, International Journal for Numerical Methods in Fluids, 60(3), 237-261, doi:10.1002/fld.1882.

Pawlowicz, R. (2018), M_Map: A mapping package for MATLAB, version 1.4j, www.eoas.ubc.ca/ $\sim$ rich/map.html.

Persson, P. O. (2006), Mesh size functions for implicit geometries and PDE-based gradient limiting, Engineering with Computers, 22(2), 95-109, doi:10.1007/s00366-006-0014-1.

Prandle, D. (2003), Relationships between tidal dynamics and bathymetry in strongly convergent estuaries, Journal of Physical Oceanography, 33(12), 2738-2750, doi:10.1175/1520-0485(2003) 033<2738:RBTDAB>2.0.CO;2.

Pringle, W. J., D. Wirasaet, A. Suhardjo, J. Meixner, J. J. Westerink, A. B. Kennedy, and S. Nong (2018a), Finite-Element Barotropic Model for the Indian and Western Pacific Oceans: Tidal Model-Data Comparisons and Sensitivities, Ocean Modelling, 129, 13-38, doi:10.1016/j.ocemod. 2018.07.003.

Pringle, W. J., D. Wirasaet, and J. J. Westerink (2018b), Modifications to Internal Tide Conversion Parameterizations and Implementation into Barotropic Ocean Models, EarthArXiv, p. 9, doi:10. 31223/osf.io/84w53.

Pringle, W. J., J. Gonzalez-lopez, B. Joyce, J. J. Westerink, and A. J. van der Westhuysen (2019), Baroclinic Coupling Improves Depth-Integrated Modeling of Coastal Sea Level Variations around Puerto Rico and the U.S . Virgin Islands, Journal of Geophysical Research: Oceans, doi: 10.1029/2018JC014682.

Remacle, J.-F., and J. Lambrechts (2016), Fast and Robust Mesh Generation on the Sphere - Application to Coastal Domains, Procedia Engineering, 163, 20-32, doi:10.1016/j.proeng.2016.11.011.

Roache, P. J. (1994), Perspective: A Method for Uniform Reporting of Grid Refinement Studies, Journal of Fluids Engineering, 116(3), 405, doi:10.1115/1.2910291.

Roberts, K. J., and W. J. Pringle (2018), OceanMesh2D: User guide - Precise distance-based two-dimensional automated mesh generation, Tech. Rep. June, University of Notre Dame, doi: 10.13140/RG.2.2.21840.61446/1.

Roberts, K. J., W. J. Pringle, and J. J. Westerink (2018), OceanMesh2D 1.0: MATLAB-based software for two-dimensional unstructured mesh generation in coastal ocean modeling, Geoscientific Model Development Discussions, p. in review, doi:10.5194/gmd-2018-203.

Sandwell, D. T., J. J. Becker, C. Olson, and A. Jackson (2014), SRTM15_PLUS: Data Fusion of SRTM Land Topography with Measured and Estimated Seafloor topography.

Shewchuk, J. R. (2002), What is a good linear finite element? - interpolation, conditioning, anisotropy, and quality measures, Tech. rep., In Proc. of the 11th International Meshing Roundtable. 
Stammer, D., R. D. Ray, O. B. Andersen, B. K. Arbic, W. Bosch, L. Carrère, Y. Cheng, D. S. Chinn, B. D. Dushaw, G. D. Egbert, S. Y. Erofeeva, H. S. Fok, J. A. M. Green, S. Griffiths, M. A. King, V. Lapin, F. G. Lemoine, S. B. Luthcke, F. Lyard, J. Morison, M. Müller, L. Padman, J. G. Richman, J. F. Shriver, C. K. Shum, E. Taguchi, and Y. Yi (2014), Accuracy assessment of global barotropic ocean tide models, Reviews of Geophysics, 52(3), 243-282, doi: 10.1002/2014RG000450.

Szpilka, C., K. Dresback, R. Kolar, J. Feyen, and J. Wang (2016), Improvements for the Western North Atlantic, Caribbean and Gulf of Mexico ADCIRC Tidal Database (EC2015), Journal of Marine Science and Engineering, 4(4), doi:10.3390/jmse4040072.

Tanaka, S., S. Bunya, J. J. Westerink, C. Dawson, and R. A. Luettich (2011), Scalability of an Unstructured Grid Continuous Galerkin Based Hurricane Storm Surge Model, Journal of Scientific Computing, 46(3), 329-358, doi:10.1007/s10915-010-9402-1.

Technology Riverside Inc., and AECOM (2015), Mesh Development, Tidal Validation, and Hindcast Skill Asessment of an ADCIRC Model for the Hurricane Storm Surge Operational Forecast System on the US Gulf-Atlantic Coast, Tech. rep., National Oceanic and Atmospheric Administration/Nation Ocean Service, Coast Survey Development Laboratory, Office of Coast Survey, doi:10.7921/G0MC8X6V.

Westerink, J. J., R. A. Luettich, A. M. Baptists, N. W. Scheffner, and P. Farrar (1992), Tide and Storm Surge Predictions Using Finite Element Model, Journal of Hydraulic Engineering, 118(10), 1373-1390, doi:10.1061/(ASCE)0733-9429(1992)118:10(1373).

Westerink, J. J., L. R. A., and M. J. C. (1994), Modelling tides in the western North Atlantic using unstructured graded grids, Tellus A, 46(2), 178-199, doi:10.1034/j.1600-0870.1994.00007.x.

Westerink, J. J., R. A. Luettich, J. C. Feyen, J. H. Atkinson, C. Dawson, H. J. Roberts, M. D. Powell, J. P. Dunion, E. J. Kubatko, and H. Pourtaheri (2008), A Basin- to Channel-Scale Unstructured Grid Hurricane Storm Surge Model Applied to Southern Louisiana, Monthly Weather Review, 136(3), 833-864, doi:10.1175/2007MWR1946.1.

White, S., and K. Hess (2016), An Assessment of the Revised VDatum for Eastern Florida, Georgia, South Carolina, and North Carolina. NOAA Technical Memorandum NOS CS 38.

Xie, D.-m., Q.-p. Zou, and J. W. Cannon (2016), Application of SWAN+ADCIRC to tide-surge and wave simulation in Gulf of Maine during Patriot's Day storm, Water Science and Engineering, 9(1), 33-41, doi:10.1016/j.wse.2016.02.003.

Xing, J., and A. M. Davies (1998), A three-dimensional model of internal tides on the MalinHebrides shelf and shelf edge, Journal of Geophysical Research: Oceans, 103(C12), 27,82127,847, doi:10.1029/98JC02149.

Xu, Q., C. Chen, J. Qi, H. Lin, R. C. Beardsley, and Y. Sun (2013), Impact of current-wave interaction on storm surge simulation: A case study for Hurricane Bob, Journal of Geophysical Research: Oceans, 118(5), 2685-2701, doi:10.1002/jgrc.20207.

Zhang, Y., and A. M. Baptista (2008), SELFE: A semi-implicit Eulerian-Lagrangian finite-element model for cross-scale ocean circulation, Ocean Modelling, 21(3-4), 71-96, doi:10.1016/j.ocemod. 2007.11.005.

Zhang, Y. J., F. Ye, E. V. Stanev, and S. Grashorn (2016), Seamless cross-scale modeling with SCHISM, Ocean Modelling, 102, 64-81, doi:10.1016/j.ocemod.2016.05.002.

Zheng, L., R. H. Weisberg, Y. Huang, R. A. Luettich, J. J. Westerink, P. C. Kerr, A. S. Donahue, G. Crane, and L. Akli (2013), Implications from the comparisons between two- and three- dimensional model simulations of the Hurricane Ike storm surge, Journal of Geophysical Research C: Oceans, 118, 3350-3369, doi:10.1002/jgrc.20248. 